\title{
Diversidad de musgos en Cuba Oriental
}

\author{
Diversity of the mosses of eastern Cuba
}

\author{
Ángel Motito Marín ${ }^{1} \&$ María Elena Potrony Hechavarría ${ }^{1}$
}

\begin{abstract}
Resumen
La flora de musgos de Cuba presenta su mayor diversidad en la región oriental. A partir del trabajo de campo, de revisiones bibliográficas y de materiales de herbarios se realiza un inventario de los musgos registrados para Cuba Oriental, reconociéndose 354 taxones infragenéricos pertenecientes a 153 géneros y 47 familias. Se registra la familia Hedwigiaceae por primera vez para Cuba, así como los géneros Braunia y Leucophanes; además de las especies Braunia squarrulosa (Hampe) Müll. Hal., Callicostella rivularis (Mitt.) A. Jaeger, Leucophanes molleri Müll. Hal., Pilotrichum bipinnatum (Schwägr.) Brid., Phyllogonium viscosum (P. Beauv.) Mitt., Pogonatum campylocarpum (Müll. Hal.) Mitt., Rhamphidium borinquense H. A. Crum \& Steere y Sphagnum portoricense Hampe.
\end{abstract}

Palabras claves: Cuba Oriental, Diversidad, Musgos.

\begin{abstract}
The mosses of Cuba have their greatest diversity in the eastern region. Based on field work, published revisions and herbarium material, an inventory of the mosses recorded for eastern Cuba recognizes 354 infrageneric taxa belonging to 153 genera and 47 families. The Hedwigiaceae family is reported for the first time from Cuba as well as the genera Braunia and Leucophanes and, the species Braunia squarrulosa (Hampe) Müll. Hal., Callicostella rivularis (Mitt.) A. Jaeger, Leucophanes molleri Müll. Hal., Pilotrichum bipinnatum (Schwägr.) Brid., Phyllogonium viscosum (P. Beauv.) Mitt., Pogonatum campylocarpum (Müll. Hal.) Mitt., Rhamphidium borinquense H. A. Crum \& Steere and Sphagnum portoricense Hampe.

Key words: Diversity, Eastern Cuba, Mosses.
\end{abstract}

\section{Introducción}

Los primeros trabajos sobre los musgos cubanos de que se tienen referencia son los de Lehmann (1834) y Sullivant (1861), este último autor en sus "Musci Cubensis" publica algunos de los musgos colectados por C. Wright en la región oriental de Cuba.

En la primera mitad del siglo XX sobresalen los estudios de Williams (1919, 1921), Britton (1922), León (1933), Thériot (1939, 1940, 1941) y Welch (1950), donde se listan y describen especies de musgos cuyos nombres hoy en día son válidos.

Hacia la segunda mitad de ese mismo siglo se publican los trabajos de Bizot (1965, 1973), Welch (1969), Schubert (1978), Duarte (1982a, b, 1995, 1997) y Zündorf (1989).

Sin lugar a dudas el estudio más completo sobre la flora de musgos cubana es el realizado por Duarte (1997) en el que se analiza de forma crítica los estudios de los briólogos que lo precedieron, indicando la presencia en nuestro territorio nacional de 396 taxones infragenéricos agrupados en dos subclases, 12 órdenes, 38 familias y 147 géneros. En este estudio se presentan, además, datos generales sobre la distribución y sustratos de algunas de las muestras examinadas. Es necesario señalar que Duarte no visitó la región oriental de Cuba; las colectas fueron realizadas por otros botánicos no especialistas en la materia, principalmente por A. Borhidi, O. Muñiz y S. Vázquez.

Lamentablemente el estudio realizado por Duarte culminó en el año 1980 (comunicación personal D. Reyes, 1992) y no fue publicado hasta el 1997, por lo que muchas de las especies que se tratan presentan problemas nomenclaturales. 


\section{Materiales y Métodos}

En este trabajo se define como región oriental los territorios de las actuales provincias de Holguín, Granma, Santiago de Cuba y Guantánamo, según la División Político-Administrativa de 1975 que es la que se sigue en los estudios de la Flora de la República de Cuba.

De 1987 al 2006 se realizaron trabajos de campo como parte de los proyectos "Diversidad biológica de los macizos montañosos Sierra Maestra y Nipe-Sagua-Baracoa", "Caracterización briológica y pteridológica del Parque Nacional Pico Cristal" y "Acciones de Educación Ambiental para el Desarrollo Sostenible en Áreas de Pluvisilva", entre otros. El muestreo se realizó principalmente en algunas zonas poco conocidas como: Alto de Iberia, El Toldo, La Melba, Meseta del Guaso y Altiplanicie de Nipe, entre otras.

Las colectas se realizaron de forma intensiva a orillas y talud de caminos, cañadas y en el interior del bosque, teniendo en cuenta los microhábitats donde puede crecer este grupo de plantas.

Se asume el ordenamiento taxonómico propuesto por Goffinet \& Shaw (2010).

Para las actualizaciones nomenclaturales se siguen las siguientes obras: Index Muscorum (Wijk et al. 1959-1969), A Dictionary of Mosses (Crosby \& Magill 1977) and Index of Mosses (Crosby et al. 1992). También se consultaron a Aguirre \& Rangel (2005), Allen \& Crosby (1986a,b), Ardiles et al. (2008), Buck \& Ireland (1985), Buck (1984, 1987a,b, 1988, 1989a,b, 1991, 1994a,b, 1998), Bytebier \& Chuat-Petiot (2007), Churchill \& Linares (1995), Churchill \& Salazar Allen (2001), Crum \& Buck (1994), Crum (1972, 1994), Dauphin \& Grayum (2005), Frahm (1987, 1991), Holz et al. (2002), Ireland (1986, 1992), Koponen (1981), Lewis et al. (2004), Magill (1994), Manuel (1994), McFarland (1994), Menzel (1985), Merwin et al. (2001), Nishimura \& Ando (1994), Ochi (1980, 1981, 1994), Pursell (1994, 1999 , 2007), Pursell et al. (1992), Reese (1993), Salazar Allen (1992), Smith (1994), Šoltes (2000), SpessardSchueth (1994), Streimann (1993) y Zander (1981, 1983, 1993, 1994).

Para los nombres de los autores de los taxones se siguió a Brummitt \& Powell (1992).

Se consultaron, además, muestras de los herbarios BSC, HAC, HAJB, JBSD, MAPR, PMA, SCZ y USD. Todos los acrónimos de los herbarios se corresponden según Holmgren et al. (1990) y Holmgren \& Holmgren (2001).
Todo el material colectado esta depositado en la Sección de Briofitas del BSC.

\section{Resultados y Discusión}

Se identificaron 3745 muestras pertenecientes a las colecciones del BSC, HAC y HAJB; se obtuvo información de 6339 ejemplares presentes en herbarios, además, de los citados en la literatura, principalmente León (1933), Thériot (1939, 1940, 1941), Bizot (1965, 1973), Welch (1969), Schubert (1978), Zündorf (1989) y Duarte (1997); realizadas en más de 500 localidades de Cuba Oriental.

En la confección de la lista final de los musgos presentes en la zona de estudio se analizaron detalladamente los trabajos de Duarte (1997) (los estudios precedentes fueron incluídos en este estudio) y Zündorf (1989), detectándose que casi el $47 \%$ de los taxones citados por ambos autores necesitaban actualización nomenclatural.

Después de realizadas las actualizaciones nomenclaturales se reconoce la presencia de 14 órdenes de musgos presentes en Cuba Oriental, 47 familias $(95.3 \%), 153$ géneros $(92.3 \%$ ) y 354 taxones infragenéricos $(87.6 \%)$. Este resultado difiere de los 218 y 318 taxones infragenéricos reconocidos por Duarte (1997) y Zündorf (1989), respectivamente, y se debe al aumento de los estudios taxonómicos y florísticos locales, regionales o nacionales realizados en el Neotrópico en las últimas décadas.

Los órdenes mejor representados en cuanto al número de familias que agrupan resultaron ser Hypnales (21), continuando en forma decreciente, Dicranales (7) y Hookeriales (5); mientras el resto de los órdenes tienen una o dos familias solamente.

Se reconoce por primera vez para Cuba la familia Hedwigiaceae.

Las familias que presentan los mayores totales de géneros son: Pottiaceae (16), Pilotrichaceae (11), Neckeraceae (9), Brachytheciaceae (8), Dicranaceae, Hypnaceae y Meteoriaceae (8), Pterobryaceae y Sematophyllaceae (6), y Bryaceae y Pylaisiadelphaceae (5). El resto de las familias tienen entre uno y cuatro géneros; existen, además, 18 familias (38.2\%) monogenéricas o que están representadas en Cuba Oriental por un solo género. Algunas familias poseen muy pocos géneros y gran cantidad de taxones infragenéricos: Calymperaceae (4 y 26), Leucobryaceae (4 y 19), Orthotrichaceae (3 y 17), Bartramiaceae (3 y 10), Fissidentaceae (1 y 30) y Sphagnaceae (1 y 10). 
Se registran dos nuevos géneros para Cuba: Braunia y Leucophanes. El género Braunia (Hedwigiaceae) con la especie Braunia squarrulosa (Hampe) Müll. Hal. se caracteriza por presentar hojas sin costa, células laminales cortorectangulares a elípticas, papilosas con paredes sinuosas, y cápsula sin perístoma. Esta familia se localiza exclusivamente en la Sierra Maestra, Gran Piedra. Los representantes de esta familia se distribuyen principalmente en las áreas montañosas de las regiones tropicales del mundo (De Luna \& Buck 1991).

El otro nuevo género mencionado anteriormente es Leucophanes (Calymperaceae) representado por la especie Leucophanes molleri Müll. Hal. Estas plantas se caracterizan por presentar una costa simple y delgada, lámina multiestratificada y en corte transversal los clorocistes se encuentran agrupados cerca del centro, hacia la superficie adaxial. Solamente se ha colectado en La Melba, en las márgenes del Río Palmares, Holguín, a los 160 m de altitud. Salazar Allen (1993a,b) señala que se puede encontrar desde el nivel del mar hasta los $800 \mathrm{~m}$ de altitud. La muestra observada de Cuba no posee esporofito, ni órganos sexuales; referente a este aspecto Salazar Allen (1993b) indica que estos musgos son dioicos y que en las poblaciones neotropicales se desconocen las plantas masculinas y los esporofitos, mientras que en las poblaciones africanas se presentan las plantas de ambos sexos y los esporofitos.

Se registran por primera vez para Cuba Oriental los siguientes géneros de musgos: Braunia, Brymela, Donnellia, Leucophanes, Pleurochaete, Pseudotrachypus y Rauiella.

Se reconocen por primera vez para Cuba las siguientes especies: Braunia squarrulosa (a su vez primer registro para las Antillas Mayores), Callicostella rivularis (Mitt.) A. Jaeger, Leucophanes molleri, Phyllogonium viscosum (P. Beauv.) Mitt., Pilotrichum bipinnatum (Schwägr.) Brid., Pogonatum campylocarpum (Müll. Hal.) Mitt., Rhamphidium borinquense H. A. Crum \& Steere y Sphagnum portoricense Hampe.

El total de taxa infragenéricos en Cuba Oriental asciende a 354, que representa el $87.6 \%$ del total de la flora de musgos de Cuba; se indican además, 34 nuevos registros para la región de estudio.

\section{Lista anotada de los musgos presentes en Cuba Oriental}

*Nuevos registros para la zona de estudio

\section{AMBLYSTEGIACEAE}

Anacamptodon cubensis (Sull.) Mitt. SANTIAGO DECUBA, El Avispero, 150-250 m, 8.X.1997, Potrony 15176 (BSC).

Campylium chrysophyllum (Brid.) Lange. SANTIAGO DE CUBA, Pico Turquino, 900-1525 m, 10.VI.1936, Acuña 289 (HAC).

\section{ANOMODONTACEAE}

Anomodon attenuatus (Hedw.) Hüb. GUANTÁNAMO, Meseta del Guaso, Limonar, cerca del sumidero del Río Cuzco, 310-321 m, 15.XII.1994, Motito y Potrony 13238 (BSC).

*Anomodon rostratus (Hedw.) Schimp. GUANTÁNAMO, Meseta de Maisí, Quemados, 200 m, 14.IV.1998, Motito y Vicario 15533 (BSC).

Schwetschkeopsis fabronia (Schwaegr.) Broth. Registro de Buck (1998).

\section{BARTRAMIACEAE}

Breutelia jamaicensis (Mitt.) A. Jaeger. GRANMA, camino a Pino del Agua, 1200 m, 13.XII.1988, Motito y Vicario 9388 (BSC). SANTIAGO DE CUBA, Sierra Cristal, zona de Corea, 500 m, 9.IV.1976, Borhidi 2925 (HAC).

Breutelia scoparia (Schwäegr.) A. Jaeger. GRANMA, Pico Bayamesa, 1700 m, 15.XI71986, Mustelier y Vicario 7499 (BSC).

Breutelia tomentosa (Brid.) A. Jaeger. GUANTÁNAMO, Alto de Iberia, camino a La Laguna, 540-600 m, 1.III.1998, Motito 15874 (BSC). SANTIAGO DE CUBA, Pico Turquino, Paso de Las Angustias, $1700 \mathrm{~m}$, 9.XII.1969, Borhidi et al. 458 (HAC).

Leiomela bartramioides (Hook.) Paris. GRANMA, Pico Regino, 1600 m, 20.IV.1979, Pócs 9091/B (BSC). SANTIAGO DE CUBA, Sierra Maestra, entre arroyos Peladero e Indio, 1000-1500 m, 27.XI.1959, López Figueiras 376 (HAC).

Leiomela filifolia Thér. GUANTÁNAMO, Baracoa, Arroyo Blanco, 160 m, 19.V.1991, Musteliery Vicario 12267 (BSC).

*Philonotis elongata (Dism.) H.A. Crum \& Steere. SANTIAGO DE CUBA, Gran Piedra, 1000-1200 m, 4.IV.1982, Buck 7661 (BSC, HAC). 
Philonotis glaucescens (Hornsch.) Broth. GUANTÁNAMO, Yunque deBaracoa, 450-540m, 1943, Carabia 480 (HAC). SANTIAGO DE CUBA, Alto de Villalón, 500 m, 9.IV.1982, Buck 9801 (BSC, HAC).

Philonotis gracillima Ångstr. GUANTÁNAMO, Yunque de Baracoa, 450-540 m, 2.I.1960, Alaín y López Figueiras 7240 (HAC).

Philonotis sphaerocarpa (Hedw.) Brid. SANTIAGO DECUBA, Gran Piedra, 1130 m, 10.IX.1978, Pócs y Reyes 9047/AD (BSC).

Philonotis uncinata (Schwägr.) Brid. GRANMA, camino a Pino del Agua, 1200 m, 13.XII.1988, Motito y Vicario 8936 (BSC). SANTIAGO DE CUBA, Gran Piedra, 1150m, 10.XI.1978, Pócs y Reyes 9046/AK(BSC).

\section{BRACHYTHECIACEAE}

Brachythecium ruderale (Brid.) W. R. Buck. GUANTÁNAMO, Sierra del Purial, norte de Puriales de Caujerí, al sur del Río Toa, 630 m, 21.X.1980, Pócs y Reyes 9186/L (BSC). SANTIAGO DECUBA, Meseta Santa María del Loreto, orillas de arroyos, 520 m, 18.V.1979, Pócs 9108/AF (BSC).

Eurhynchium pulchellum (Hedw.) Jenn. Registro de Buck (1998).

Helicodontium capillare (Hedw.) A. Jaeger. GUANTÁNAMO, Baracoa, Duaba, Santa Rosa del Duaba, 50 m, 27.XI.1978, Pócs y Reyes 9056/D (BSC). SANTIAGODECUBA, Alto de Villalón, Río Ramona, 500 m, 9.IV.1982, Shaw 5386 (BSC, HAC).

Meteoridium remotifolium (Müll. Hal.) Manuel. GRANMA, La Bayamesa, Arroyo Nuevo Mundo, 1000-1050 m, 15.XII.1988, Motito y Vicario 8808 (BSC). SANTIAGO DE CUBA, entre Pico Cuba al Pico Turquino, 1800-1972 m, 14.IV.1987, Mustelier y Vicario 9506 (BSC).

Palamocladium leskeoides (Hook.) E. Britton. GRANMA,LomaLaSabina, 1100-1200m, 19.IV.1979, Pócs y Duany 9085/AV (BSC). GUANTÁNAMO, Baracoa, Arroyo Blanco, 160 m, 12.V.1991, Mustelier y Vicario 12167 (BSC).

*Rhynchostegium robustum W.R. Buck. GUANTÁNAMO, Meseta del Guaso, Limonar, cerca del sumidero del río Cuzco, 400 m, 15.XII.1994, Motito y Potrony 13231 (BSC).

Rhynchostegium scariosum (Taylor) A. Jaeger. Registro de Buck (1998).

Rhynchostegium serrulatum (Hedw.) A. Jaeger. GUANTÁNAMO, Meseta de Maisí, Quemados, 200 m, 14.IV.1998, Motito y Vicario 15525 (BSC). SANTIAGO DE CUBA, Gran Piedra, $1100 \mathrm{~m}$, 3.IV.1982, Buck 7571 (BSC, HAC).
*Squamidium isocladum (Renauld. \& Cardot) Broth. SANTIAGO DE CUBA, Sierra Maestra, Gran Piedra, 1000 m, 6.IV.1982, Buck s/n (BSC, HAC).

Squamidium leucotrichum (Taylor) Broth. GRANMA, Pico Bayamesa, 1500-1700 m, 17.XII.1988, Motito y Vicario 9848 (BSC). SANTIAGO DE CUBA, Pico Suecia, 1700-1750 m, 6.XII.1969, Muñiz 443 (HAC).

*Squamidium livens (Schwägr.) Broth. SANTIAGO DE CUBA, Gran Piedra, 1000-1100 m, 2.IV.1982, Shaw 5332 (BSC, HAC).

Squamidium nigricans (Hook.) Broth. GRANMA, subida a Pico Bayamesa, 1500-1700 m, 17.XII.1988, Motito y Vicario 9811 (BSC). SANTIAGO DECUBA, Gran Piedra, 1200 m, 10.III.1989, Reyes 10438(BSC). Zelometeorium patulum (Hedw.) Manuel. GRANMA, La Bayamesa, Nuevo Mundo, 1100 m, 15.XII.1988, Motito y Vicario 8812 (BSC). SANTIAGODECUBA, Gran Piedra, La Isabelica, 1000-1100 m, 21.IV.1987, Motito 8685 (BSC).

\section{BRUCHIACEAE}

Trematodon longicollis Michx. GRANMA, sur de Santo Domingo, valle río Yara, 300 m, 14.IV.1979, Pócs y Duany 9072/T (BSC).

\section{BRYACEAE}

Acidodontium megalocarpum (Hook.) Renauld. \& Cardot. SANTIAGO DE CUBA, Pico Turquino, 1370-1700, 10.VI.1936, Acuña 410 (HAC).

Anomobryum filiforme (Dicks.) Solms. var. filiforme. SANTIAGO DE CUBA, Sierra Maestra, entre arroyos Peladero e Indio, 1000-1500 m, 27.XI.1959, López Figueiras 421 (HAC).

*Brachymenium globosum A. Jaeger. SANTIAGO DE CUBA, Pico Suecia, 1700-1750 m, 7.XII.1969, Muñiz 316 (HAC).

Brachymenium speciosum (Hook. f. \& Wilson) Steere. GRANMA, Pico Regino, 1600 m, 20.IV.1979, Pócs 9091/AD (BSC). SANTIAGO DECUBA, Gran Piedra, 1150 m, 2.XII.1970, Borhidi et al. 1061 (HAC).

Brachymenium wrightii (Sull.) Broth. GUANTÁNAMO, Yunque de Baracoa, 450-540 m, 10.XI.1983, Mustelier 10747 (BSC). SANTIAGO DE CUBA, Santa Ana, cerca de Santiago de Cuba, 500 m, 23.III.1902, Hamilton 80 (HAC).

Bryum argenteum Hedw. GUANTÁNAMO, Meseta del Guaso, La Prenda, 250 m, 1933, Hioram 218 (HAC). SANTIAGO DE CUBA, Gran Piedra, 1000-1200 m, 25.IX.1970, Reyes 5063 (BSC). 
Bryum billardieri Schwägr. GUANTÁNAMO, Piedra La Vela, 500-600 m, 11.XII.1997, Motito et al. 15782 (BSC). SANTIAGO DE CUBA, Gran Piedra, 1000-1200 m, 4.IV.1982, Buck 7649 (BSC, HAC).

Bryum capillare Hedw. HOLGUÍN, Sierra de Nipe, sur del corte de leña, 600-700, 16.X.1941, Morton y Acuña 3910 (HAC).

Bryum coronatum Schwägr. SANTIAGO DECUBA, Morro de Santiago de Cuba, cuevas en la ladera norte, 30 m, 08.V.1979, Pócs 9100/C (BSC). Sierra del Cristal, camino a Pico Cristal, 810 m, 21.III.2001, Motito y Trapero 17047 (BSC).

Bryum hioramii Thér. SANTIAGODECUBA, Lomas San Juan, 900 m, 1940, Hioram 11801 (HAC).

Bryum leonii Thér. GUANTÁNAMO, Meseta de Maisí, Los Llanos, 420 m, 12.VIII.1938, León 17117 (HAC).

*Bryum limbatum Müll. Hal. SANTIAGO DE CUBA, Alto de Villalón, 575 m, 16.I.1985, Echezarreta 5882 (BSC).

*Bryum procerum A. Schimp. ex Besch. SANTIAGO DE CUBA, Alto de Villalón, 300 m, 6.X.1980, Pócs y Caluff 9163/F (BSC).

Bryum pseudocapillare Besch. GUANTÁNAMO, Meseta del Guaso, La Prenda, 250 m, 1924, Hioram 217 (HAC).

*Bryum renauldii Röll. ex Renauld. \& Cardot. GUANTÁNAMO, Meseta del Guaso, Limonar, cerca del sumidero del Río Cuzco, 310-320 m, 15.XII.1994, Motito y Potrony 13223 (BSC).

Rhodobryum beyrichianum (Hornsch.) Müll. Hal. ex Hampe. GUANTÁNAMO, Yunque de Baracoa, 450-540 m, 17.XII.1985, Mustelier 7870 (BSC). HOLGUÍN, Sierra de Nipe, Cuchuflí, 640-720 m, 30.IV.1997, Motito y Vicario 14922 (BSC).

\section{CALYMPERACEAE}

Calymperes afzelii Sw. GUANTÁNAMO, Meseta de Maisí, Quemados, 200 m, 12.IV.1998, Motito y Vicario 15503 (BSC). SANTIAGO DE CUBA, Meseta Santa María del Loreto, norte de Yerba de Guinea, 240 m, 27.X.1980, Pócs y Caluff 9194/ $G$ (BSC).

Calymperes erosum Müll. Hal. GUANTÁNAMO, Yunque de Baracoa, 200-450 m, 10.XI.1983, Mustelier y Vicario 15494 (BSC).

Calymperes guildingii Hook. \& Grev. GUANTÁNAMO, Baracoa, Duaba, El Dajao, 460-480 m, 11.X.1991, Mustelier y Vicario 12786 (BSC).
*Calymperes levyanum Besch. GUANTÁNAMO, Yunque de Baracoa, 200-450 m, 26.XI.1978, Pócs y Reyes 9063/AM (BSC). SANTIAGO DE CUBA, Sierra Cristal, Pico Cristal, 1231 m, 19.II.1976, Catasús 2748 (HAC).

Calymperes lonchophyllum Schwägr. HOLGUÍN, Moa, La Melba, cerca de la Cascada Dos Comadres, 400 m, 10.X.1980, Pócs y Reyes 9076/AC (BSC). SANTIAGO DE CUBA, Loma del Gato, $1100 \mathrm{~m}$, 25.IX.1936, Acuña 9749 (HAC).

Calymperes palisotii Schwägr. HOLGUÍN, Silla de Gibara, 100m,22.X.1978,Pócs 9037/A(BSC).SANTIAGO DE CUBA, La Lata, Brazo Escondido, sur de Filé, 400-500 m, 24.X.1969, Borhidi et al. 185e (HAC).

*Calymperes pallidum Mitt. HOLGUÍN, alrededores de Candelaria Munilla, 50 m, 23.X.1978, Pócs 9040/ A (BSC).

*Calymperes tenerum Müll. Hal. GUANTÁNAMO, Meseta del Guaso, Limonar, cerca del sumidero del Río Cuzco, 400 m, 15.XII.1994, Motito y Potrony 13244 (BSC).

Leucophanes molleri Müll. Hal. HOLGUÍN, Moa, La Melba, márgenes del Río Palmares, 160 m, 20.IX.1997, Potrony 15283 (BSC).

Octoblepharum albidum Hedw. GUANTÁNAMO, Baracoa, punta barlovento de la Bahía de Taco, 1020 m, 6.III.1998, Motito 15844 (BSC). HOLGUÍN, Cerro Galano, sur de Melones, Arroyo Sao Redondo, 200 m, 18.X.1978, Pócs y Catasús 9024/A (BSC). SANTIAGO DE CUBA, La Alcarraza, 600 m, 25.I.1989, Motito 9478 (BSC).

Octoblepharum cocuiense Mitt. GUANTÁNAMO, Yunque de Baracoa, 450-540 m, 26.XI.1978, Pócs y Reyes 9066/R (BSC). HOLGUÍN, Sierra Cristal, Arroyo Cirino, 220 m, 31.III.2004, Motito 18180 (BSC).

Octoblepharum erectifolium Mitt. ex R.S. Williams. HOLGUÍN, Sierra de Nipe, Pinares de Mayarí, 400 m, 27.III.2004, Motito 18187 (BSC). SANTIAGO DE CUBA, Sierra Cristal, primera cañada después de Batista, 770 m, 25.III.2001, Motito 16991 (BSC).

Octoblepharum pulvinatum (Dozy \& Molk.) Mitt. GUANTÁNAMO, Baracoa, Duaba, Arroyo Jaragual, 250-280 m, 14.X.1991, Musteliery Vicario 12706(BSC).

*Syrrhopodon africanus subsp. graminicola (R.S. Williams) Reese. HOLGUÍN, Sierra de Nipe, Woodfred, 450-550 m, s.d., Shafer 3732a (HAC).

Syrrhopodon elongatus Sull. var. elongates. HOLGUÍN, Moa, La Melba, cerca de la Cascada Dos Comadres, 400 m, 10.X.1980, Pócs y Reyes 9170/D (BSC). SANTIAGO DE CUBA, Sierra Cristal, Pico Cristal, 1231 m, 28.III.2001, Trapero 17027 (BSC). 
Syrrhopodon gaudichaudii Mont. GRANMA, Sierra Maestra, entre Pico Joaquín y Pico Regino, 1600 m, 20.IV.1979, Pócs 9090/J (BSC). SANTIAGO DE CUBA, Pico Palma Mocha, 1200-1300 m, 16.IV.1979, Pócs y Duany 9081/C (BSC).

Syrrhopodon incompletus Schwägr. var. incompletus. HOLGUÍN, Sierra de Nipe, Cuchuflí, 640-720 m, 30.IV.1997, Motito y Vicario 14906 (BSC). SANTIAGO DE CUBA, Sierra Cristal, cabezadas del Río Miguel, Arroyo Manzano, 680 m, 24.III.2001, Motito et al. 17166 (BSC).

Syrrhopodon incompletus var. berteroanus (Brid.) Reese. GUANTÁNAMO, Piedra La Vela, 600 m, 05.XII.1997, Motito et al. 15690 (BSC). SANTIAGO DE CUBA, Sierra Cristal, Arroyo Manzano, entre La China y Batista, 600-650 m, 10.XI.1989, Motito et al. 10219 (BSC).

Syrrhopodon leprieurii Mont. SANTIAGODECUBA, Pico Turquino, 900-1525m, 10.VI.1936, Acuña s/n(HAC).

Syrrhopodon lycopodioides (Brid.) Müll. Hal. GRANMA, Pico Joaquín, 1400 m, 20.IV.1979, Pócs 9088/O (BSC). SANTIAGO DE CUBA, Sierra Cristal, camino a La Zanja, 680 m, 24.III.2001, Motito et al. 17008 (BSC). Syrrhopodon parasiticus (Brid.) Besch. HOLGUÍN, Moa, La Melba, cerca de la Cascada Dos Comadres, 400 m, 10.X.1980, Pócs y Reyes 9170/BS (BSC). SANTIAGO DE CUBA, Gran Piedra, Jardín La Siberia, 1000-1100 m, 21.V.1979, Pócs y Duany 9115/ CY(BSC).

*Syrrhopodon prolifer Schwägr. var. prolifer. GUANTÁNAMO, Reserva Cupeyal del Norte, 500600m, 28.VIII.1986, Mustelier 7974(BSC). SANTIAGO DE CUBA, Sierra Cristal, Arroyo Manzano, entre La China y Batista, 600-650 m, 10.XI.1989, Motito et al. 10251 (BSC).

Syrrhopodon prolifer var. acanthoneuros (Müll. Hal.) Müll. Hal. GUANTÁNAMO, Baracoa, Arroyo Blanco, 160 m, 12.V.1991, Mustelier y Vicario 12101 (BSC). SANTIAGO DE CUBA, Sierra Cristal, camino a Canadá, 650 m, 15.II.1989, Motito et al. 10502 (BSC).

Syrrhopodon prolifer var. cincinnatus (Hampe) Reese. HOLGUÍN, Moa, La Melba, cerca de la Cascada Dos Comadres, 400 m, 10.X.1980, Pócs y Reyes 9170/ $B X$ (BSC). SANTIAGO DE CUBA, Sierra Cristal, camino a Pico Cristal, 810 m, 21.III.2001, Motito y Trapero 16939 (BSC).

Syrrhopodon prolifer var. scaber (Mitt.) Reese. GUANTÁNAMO, Meseta de Maisí, Quemados, 200m, 13.IV.1998, Motito y Vicario 15492 (BSC). HOLGUÍN, Sierra de Nipe, Cuchuflí, 720 m, 28.IV.1997, Motito y Vicario 14824 (BSC).
Syrrhopodon prolifer var. tenuifolius (Sull.) Reese. GUANTÁNAMO, Piedra La Vela, 600 m, 8.XII.1997, Motito et al. 15821 (BSC). HOLGUÍN, Gibara, Loma de Candelaria, 50-100 m, 23.X.1978, Pócs 9039/T(BSC).

\section{CRYPHAEACEAE}

Cryphaea filiformis (Hedw.) Brid. GRANMA, márgenes del Arrroyo Barrio Nuevo, 15.V.1988, Panfet 64677(HAJB). SANTIAGO DE CUBA, Gran Piedra, 1200 m, 06.IV.1982, Buck 7746 (BSC, HAC).

Schoenobryum concavifolium (Griff.) Gangulee. GUANTÁNAMO, Meseta del Guaso, Limonar, camino hacia El Fomento, 300-400 m, 16.XII.1994, Motito y Potrony 13307(BSC). SANTIAGODECUBA, Sierra Cristal, Mayarí Arriba, frutales del Museo del Segundo Frente, 175 m, 7.XI.1978, Pócs et al. 9043/A (BSC).

\section{DALTONIACEAE}

Adelothecium bogotense (Hampe) Mitt. SANTIAGO DE CUBA, entre Pico Palma Mocha y las Lagunitas, 1200-1300 m, 16.IV.1979, Pócs y Duany 9079/T(BSC).

Daltonia longifolia Taylor. GRANMA, Loma Subida Albear, cerca delPico Bayamesa, 1300-1400m, 3.XI.1978, Pócs 9066/DF (BSC). SANTIAGO DE CUBA, Gran Piedra, 1150m, 10.XI.1978, Pócs y Reyes 946/BH(BSC).

Daltonia stenophylla Mitt. SANTIAGO DE CUBA, Gran Piedra, 1000-1200 m, 4.IV.1982, Buck 7647(BSC).

Leskeodon auratus (Müll. Hal.) Broth. Registro de Buck (1998).

Leskeodon andicola (Mitt.) Broth. GUANTÁNAMO, Sierra del Purial, Loma La Farola, 480 m, 25.XI.1978, Pócs y Reyes 9062/L (BSC). HOLGUÍN, Reserva de Jaguaní, Río Anacleto, 200-400 m, 17.II.1970, Borhidi et al. 718 a (HAC).

Leskeodon cubensis (Mitt.) Thér. GRANMA, Loma Subida Albear, cerca del Pico Bayamesa, 1300-1400 m, 30.XI.1978, Pócs y Catasús 9066/BA (BSC). SANTIAGO DE CUBA, Gran Piedra, 1050 m, 17.XI.1978, Pócs y Reyes 9057/DU (BSC).

\section{DICRANACEAE}

Aongstroemia jamaicensis Müll. Hal. GRANMA, Pico Bayamesa, 1300-1500 m, 17.XII.1988, Motito y Vicario 8881 (BSC).

Dicranella brachyblepharis (Müll. Hal.) Mitt. SANTIAGO DE CUBA, Gran Piedra, 1050 m, 17.XI.1978, Pócs y Reyes 9057/BK (BSC).

Dicranellaharrisii (Müll.Hal.)Broth. GUANTÁNAMO, Reserva Cupeyal del Norte, 500-600 m, 9.II.1970, Borhidi et al. 734 (HAC). SANTIAGODECUBA, Gran Piedra, 1000 m, 4.IV.1982, Shaw 5145 (BSC, HAC). 
Dicranella hilariana (Mont.) Mitt. SANTIAGO DE CUBA, Alto de Villalón, Río Ramona, 500 m, 9.IV.1982, Buck 7804 (BSC, HAC).

Dicranella hioramii (Thér.) Duarte var. hioramii. HOLGUÍN, Reserva de Jaguaní, La Melba, 15.II.1970, Borhidi et al. 742 (HAC). SANTIAGO DE CUBA, Gran Piedra, cañada La Siberia, 1000 m, 6.II.1988, Mustelier 8826 (BSC).

Dicranella hioramii var. acunae (Thér.) Duarte. SANTIAGO DE CUBA, Loma del Gato, 900 m, s.d., Acuña 167 (HAC).

Dicranella longirostris (Schwägr.) Mitt. SANTIAGO DECUBA, Loma del Gato, minas del Cobre, 7.VIII.1921, León et al. 10363 (HAC).

Dicranella reticulata (Müll. Hal.) Paris. SANTIAGO DECUBA, El Cobre, 21.II.1902, Pollard y Palmer 428 (HAC). Gran Piedra, 1000 m, 2.IV.1982, Shaw 5035 (BSC, HAC).

Dicranella vaginata (Hook.) Cardot. GRANMA, Sierra Maestra, Pino del Agua, 1800 m, 12.XII.1988, Motito y Vicario 9389 (BSC). SANTIAGODECUBA, Gran Piedra, Jardín La Siberia, 1000 m, 19.II.1987, Motito 4780 (BSC).

Dicranella varia (Hedw.) Schimp. HOLGUÍN, Sierra de Nipe, Loma La Mensura, 800 m, 8.IX.1979, Borhidi 6328 (HAC).

Eucamptodontopsis pilifera (Mitt.) Broth. GUANTÁNAMO, Alto de Iberia, afluente del Río Macaganigua, 20.I.2002, Sánchez et al. 79265 (HAJB). HOLGUÍN, Revuelta de Los Chinos, 850-980 m, 12.X.1980, Pócs 9175/AJ (BSC).

Holomitrium calycinum (Hedw.) Mitt. GRANMA, Sierra Maestra, pico al suroeste del poblado Pino del Agua, 1440 m, 01.XI.1978, Pócs 9067/AN(BSC). SANTIAGO DE CUBA, Alto de Villalón, 575 m, 16.I.1985, Mustelier 4788 (BSC).

Leucoloma albulum (Sull.) A. Jaeger. HOLGUÍN, Sierra de Nipe, Loma La Mensura, 200 m, 13.XI.1978, Pócs y Reyes 9055/AO (BSC). SANTIAGO DE CUBA, Gran Piedra, 1000 m, 11.I.1988, Motito y Reyes 9155 (BSC).

Leucoloma cruegerianum (Müll. Hal.) A. Jaeger. HOLGUÍN, SierradeNipe,Cuchuflí,640-700m,29.IV.1997, Motito y Vicario 14802 (BSC). SANTIAGODECUBA, Sierra Cristal, primera cañada después de Batista, 770 m, 25.III.2001, Motito 16987 (BSC).

Leucoloma mariei Besch. GRANMA, Pico Martí, 1700 m, 31.III.1990, Potrony 11484(BSC). SANTIAGODE CUBA, Gran Piedra, detrás de la cafetería, $1000 \mathrm{~m}$, 11.II.2000, Motito et al. 16568 (BSC).
Leucoloma schwaneckeanum (Hampe) Broth. GRANMA, cima de Pico Bayamesa, $1700 \mathrm{~m}$, 21.VI.2003, Potrony 17848 (BSC). SANTIAGO DE CUBA, Gran Piedra, detrás de la cafetería, 1000 m, 11.II.2000, Motito et al. 16552 (BSC).

Leucoloma serrulatum Brid. GUANTÁNAMO, Piedra La Vela, 600 m, 5.XII.1997, Motito et al. 15620 (BSC). HOLGUÍN, Sierra de Nipe, La Micro Onda, 700 m, 27.IV.1997, Motito y Vicario 15202 (BSC).

Leucoloma subimmarginatum (Müll. Hal.) A. Jaeger. GUANTÁNAMO, Yunque de Baracoa, 500-540 m, 10.VII.1986, Vicario 7951 (BSC). HOLGUÍN, Moa, Altiplanicie del Toldo, 700 m, 21.IV.2001, Potrony 17516 (BSC).

Macrodictyum proliferum (Mitt.) E.H. Hegew. SANTIAGO DE CUBA, Loma del Gato, 1100 m, 9.XII.1920, Clemente 306 (HAC).

Macrodictyum wrightii (Sull.) E.H. Hegew. SANTIAGO DE CUBA, Sierra Cristal, Pico Cristal, 1131 m, 19.II.1976, Catasús s/n (HAC).

Paraleucobryum albicans (Schwägr.) Loeske. GUANTÁNAMO, Baracoa, Arroyo Blanco, 160-200 m, 18.V.1991, Mustelier y Vicario 12299 (BSC). SANTIAGO DE CUBA, Pico Palma Mocha, 1380 m, 16.IV.1979, Pócs y Duany 9083/E (BSC).

\section{DIPHYSCIACEAE}

Diphyscium foliosum (Hedw.) Mohr. SANTIAGO DECUBA, Pico Turquino, 1300-1800 m, 10.VI.1936, Acuña 376 (HAC).

\section{DITRICHACEAE}

Ditrichum rufescens (Hampe) Hampe. SANTIAGO DE CUBA, Gran Piedra, La Isabelica, 1100 m, 31.X.1980, Pócs 9202/H (BSC).

Rhamphidium borinquense H. A. Crum \& Steere. SANTIAGODE CUBA, Gran Piedra, Arroyo Negro, 1000 m, 5.IV.1982, Buck 7695 (BSC, HAC).

Rhamphidium dicranoides (Müll. Hal.) Paris. SANTIAGO DE CUBA, Gran Piedra, 1000 m, 3.IV.1982, Shaw 5152 (BSC, HAC).

\section{ENTODONTACEAE}

Entodon beyrichii (Schwägr.) Müll. Hal. GUANTÁNAMO, Meseta del Guaso, Yambeque, 550-580 m, 10.V.1995, Motito y Vicario 13462 (BSC).

Entodon macropodus (Hedw.) Müll. Hal. GUANTÁNAMO, Meseta del Guaso, Limonar, cerca del sumidero del Río Cuzco, 310-320 m, 15.XII.1994, Motito y Potrony 13237(BSC). SANTIAGODECUBA, Gran Piedra, 1200m, 6.IV.1982, Buck7754(BSC,HAC). 
Erythrodontium longisetum (Hook.) Paris. SANTIAGO DE CUBA, Gran Piedra, 1000 m, 25.III.1968, Reyes 5047 (BSC).

\section{ERPODIACEAE}

Erpodium biseriatum (Austin) Austin. SANTIAGO DE CUBA, Pico Turquino, s.d., Acuña 377 (HAC).

Erpodium domingense (Spreng.) Müll. Hal. GUANTÁNAMO, Novaliches, surde GUANTÁNAMO, 10 m, 19.IX.1921, Hioram 5134 (HAC). SANTIAGO DE CUBA, Meseta Santa María del Loreto, sobre Santa Ana, 300-500 m, 18.V.1979, Pócs 9170/A (BSC).

\section{FABRONIACEAE}

Fabronia ciliaris var. wrightii (Sull.) W. R. Buck. SANTIAGO DE CUBA, Gran Piedra, primera fuente a la carretera superior, 600-700 m, 26.X.1969, Borhidi et al. 883 (HAC).

\section{FISSIDENTACEAE}

Fissidens angustifolius Sull. SANTIAGO DE CUBA, Morro de Santiago de Cuba, 30 m, 21.X.1969, Borhidi y Muñiz 611 (HAC).

Fissidens asplenioides Hedw. GRANMA, La Bayamesa, Nuevo Mundo, El Obelisco, 1000 m, 14.XII.1988, Motito y Vicario 6142 (BSC). HOLGUÍN, Sierra de Nipe, Cuchuflí, 640-700 m, 29.IV.1997, Motito y Vicario 14867 (BSC).

Fissidens bryoides Hedw. HOLGUÍN, orillas del Río Bariay, 30m, 17.X.1978, Pócs 9020/E(BSC). SANTIAGO DE CUBA, Playa de Siboney, 20 m, 12.XI.1978, Pócs y Reyes $9051 / B$ (BSC).

Fissidens crispus Mont. SANTIAGO DE CUBA, Gran Piedra, Arroyo Negro, 1000 m, 5.IV.1982, Shaw 7698 (BSC, HAC).

Fissidens dissitifolius Sull. GUANTÁNAMO, Yunque de Baracoa, 450-540 m, 26.XI.1978, Pócs y Reyes 9064/N (BSC).

Fissidens elegans Brid. GRANMA, Loma La Sabina, 1100-1200 m, 19.IV.1979, Pócs y Duany 9085/Q (BSC). HOLGUín, Reserva de Jaguaní, Río Anacleto, 200-400 m, 17.II.1979, Borhidi et al. 718 (HAC). GUANTÁNAMO, Yunque de Baracoa, 200450 m, 26.XI.1978, Pócs y Reyes 9063/Y (BSC).

Fissidens flaccidus Mitt. GUANTÁNAMO, Meseta del Guaso, Limonar, palmar en cañada alrededor del poblado, 350 m, 16.XII.1994, Motito 13949 (BSC).

Fissidens fontanus (B.-Pyl.) Steud. GRANMA, Pico Bayamesa, nacimiento del Arroyo 26, 1450m, 20.III.1987, J. Reyes y Caluff 5391 (BSC).
Fissidens guianensis Mont. var. guianensis. Registro de Pursell (2007).

Fissidens imbricatus E. Britton \& E. B. Bartram. HOLGUÍN, Sierra de Nipe, Loma de La Bandera, 400 m, 13.XI.1978, Pócs y Reyes 9052/B (BSC). SANTIAGO DE CUBA, Sierra Cristal, Pico Cristal, 1231 m, 28.III.2001, Trapero 17038 (BSC).

Fissidens inaequalis Mitt. GRANMA, Pico Bayamesa, 1200-1300 m, 14.XI.1986, Mustelier y Vicario 5374 (BSC). GUANTÁNAMO, Yunque de Baracoa, 200450 m, 10.XI.1983, Mustelier 4744 (BSC).

Fissidens intermedius Müll. Hal. GRANMA, Loma La Sabina, 1100-1200, 19.IV.1979, Pócs y Duany 9085/YZ(BSC). SANTIAGO DECUBA, Gran Piedra, 1050 m, 17.XI.1978, Pócs y Reyes 9057/BH (BSC).

Fissidens lagenarius Mitt. var. lagenarius. Registro de Pursell (2007).

Fissidens lagenarius var. muriculatus (Mitt.) Pursell. GUANTÁNAMO, Meseta del Guaso, Limonar, detrás del Centro Científico, 400-450 m, 14.XII.1994, Motito y Potrony 13275 (BSC).

Fissidens oblongifolius Hook. f. \& Wilson. GUANTÁNAMO, Yunque de Baracoa, 450-540 m, 26.XI.1978, Pócs y Reyes 9064/S (BSC).

Fissidens pallidinervis Mitt. SANTIAGO DE CUBA, Gran Piedra, Pico Kentucky, 1000 m, 5.IV.1982, Buck 7669 (BSC, HAC).

Fissidens palmatus Hedw. GUANTÁNAMO, Meseta de Maisí, Quemados, 200 m, 12.IV.1998, Motito y Vicario 15520 (BSC). SANTIAGODECUBA, Subida a Monte Biset, 290-650m, 12.V.2004, MotitoyRivera 18227(BSC).

Fissidens pellucidus Hornsch. var. pellucidus. Registro de Pursell (2007).

Fissidens petrophilus Sull. GRANMA, cañada cerca de Pino del Agua, 1300 m, 16.XII.1988, Motito y Vicario 8888 (BSC). SANTIAGO DE CUBA, Sierra Cristal, Arroyo Manzano, entre La China y Batista, 600-650 m, 10.XI.1989, Motito y Potrony 10198(BSC). Fissidens polypodioides Hedw. GUANTÁNAMO, Viento Frío, camino de Vía Mulata a Arroyo La Pulga (La Gurbia), 747 m, 4.VI.2004, Motito et al. 18585 (BSC). SANTIAGO DE CUBA, Alto de La Francia, 13001400 m, 21.XII.1986, Musteliery Vicario 5306 (BSC).

Fissidens prionodes Mont. GUANTÁNAMO, Reserva de Jaguaní, Arroyo Bueno, 150 m, 10.X.1980, Pócs y Reyes 9169/O (BSC). HOLGUÍN, Moa, La Melba, cerca de la Cascada Dos Comadres, 400 m, 10.X.1980, Pócs y Reyes 9170/K (BSC). 
Fissidens radicans Mont. GUANTÁNAMO, Yunque de Baracoa, 500-540 m, 10.VII.1986, Vicario 7948 (BSC). SANTIAGO DE CUBA, Gran Piedra, alrededores de La Gran Sofía, 700 m, 26.III.1987, Motito 4729 (BSC).

Fissidens reticulosus (Müll. Hal.) Mitt. GUANTÁNAMO, Reserva de Jaguaní, Arroyo Bueno, 150 m, 10.X.1980, Pócs y Reyes 9169/Y (BSC). HOLGUÍN, Sierra de Nipe, Loma La Mensura, 400-500 m, 27.XI.1985, Mustelier y Vicario 4996 (BSC).

Fissidens rochensis Broth. in Urban. Registro de Pursell (2007).

Fissidens serratus Müll. Hal. var. serratus. GUANTÁNAMO, Piedra La Vela, camino al Arroyo Sonador, 550-680 m, 6.XII.1997, Motito et al. 115646 (BSC). SANTIAGO DE CUBA, Gran Piedra, 10001200 m, 7.XII.1969, Borhidi et al. 1065e (HAC).

Fissidens submarginatus Bruch. in C. Krauss. Registro de Pursell (2007).

Fissidens weirii Mitt. var. weirii. GUANTÁNAMO, Piedra La Vela, 600 m, 10.XII.1997, Motito et al. 15743 (BSC). SANTIAGODECUBA, Gran Piedra, $1150 \mathrm{~m}$, 10.XI.1978, Pócs y Reyes 9046/CH (BSC).

Fissidens weirii var. hemicraspedophyllus (Cardot) Pursell. Registro de Pursell (2007).

Fissidens yucatanensis Steere. SANTIAGODECUBA, Alto de Villalón, Finca Santa Isabel, 370 m, 18.XI.1978, Pócs y Reyes 9059/E (BSC).

Fissidens zollingeri Mont. GRANMA, Loma La Sabina, 1100-1200 m, 19.IV.1979, Pócs 9085/C (BSC). HOLGUÍN, orillas del Río Bariay, 30 m, 17.X.1978, Pócs 9020/E (BSC).

\section{FUNARIACEAE}

Entosthodon bonplandii (Hook.) Mitt. SANTIAGO DE CUBA, Gran Piedra, 1000 m, 3.IV.1982, Shaw 5122 (BSC, HAC).

Funaria hygrometrica var. calvescens (Schwägr.) Mont. SANTIAGODECUBA, entre Pico Palma Mocha y Las Lagunitas, 1200-1300 m, 16.IV.1979, Pócs y Duany 9079/R (BSC).

\section{HEDWIGIACEAE}

Braunia squarrulosa (Hampe) Müll. Hal. SANTIAGO DE CUBA, Gran Piedra, alrededores de La Mercedita, 800-1000 m, 13.III.1987, Motito 4801 (BSC).

\section{HELICOPHYLLACEAE}

Helicophyllum torquatum (Hook.) Cardot. GUANTÁNAMO, Meseta del Guaso, Loma Lagunato, ladera norte sobre Josefita, 240-400 m, 27.X.1980,
Pócs $s / n$ (BSC). SANTIAGO DE CUBA, Meseta Santa María del Loreto, norte de Yerba de Guinea, 240 m, 27.X.1980, Pócs s/n (BSC).

\section{HOOKERIACEAE}

Crossomitrium epiphyllum (Mitt.) Müll. Hal. GUANTÁNAMO, Meseta del Guaso, Limonar, cerca del sumidero del Río Cuzco, 310-320 m, 15.XII.1994, Motito y Potrony 13212 (BSC). HOLGUÍN, Sierra Cristal, Arroyo Cirino, 220 m, 30.III.2004, Motito 18181 (BSC).

Crossomitrium patrisiae (Brid.) Müll. Hal. GUANTÁNAMO, Meseta del Guaso, Yambeque, 450-480 m, 8.IV.1995, Motito y Vicario 13415 (BSC). SANTIAGO DE CUBA, Gran Piedra, La Isabelica, 1000 m, 11.II.2000, Motito et al. 16537 (BSC).

Hookeria acutifolia Hook. \& Grev. GRANMA, La Bayamesa, Nuevo Mundo, 900-1000 m, 11.XI.1986, Mustelier 7348 (BSC). SANTIAGO DE CUBA, Sierra Cristal, albergue de La China, 600-650 m, 11.XI.1989, Motito et al. 10306 (BSC).

\section{HYLOCOMIACEAE}

Ctenidium malacodes Mitt. GUANTÁNAMO, Piedra La Vela, 600 m, 8.XII.1997, Motito et al. 15824 (BSC). HOLGUÍN, Sierra de Nipe, La Micro Onda, 700 m, 26.IV.1997, Motito y Vicario 15031 (BSC).

\section{HYPNACEAE}

Chryso-hypnum diminutivum (Hampe) W. R. Buck. GRANMA, Loma Subida Albear, cerca del Pico Bayamesa, 1300-1400 m, 30.XI.1978, Pócs 9066/AH (BSC). SANTIAGODE CUBA, Gran Piedra, $1000 \mathrm{~m}$, 3.IV.1982, Shaw $s / n$ (BSC, HAC).

Ectropothecium leptochaeton (Schwägr.) W.R. Buck. GUANTÁNAMO, Meseta del Guaso, Limonar, cerca del sumidero del Río Cuzco, 400 m, 15.XII.1994, Motito y Potrony 13247 (BSC). SANTIAGO DE CUBA, Pico Turquino, Paso de Las Angustias, 1700 m, 9.XII.1969, Borhidi et al. 3041 d (HAC).

Hypnum polypterum (Mitt.) Broth. GUANTÁNAMO, Yunque de Baracoa, 200-450 m, 26.XI.1978, Pócs y Reyes 9063/K (BSC). SANTIAGO DE CUBA, Pico Palma Mocha, 1380 m, 16.IV.1979, Pócs y Duany 9083/J (BSC).

Mittenothamnium reptans (Hedw.) Cardot. GRANMA, LomaLaSabina, 1100-1200m, 19.IV.1979, Pócs y Duany 9085/AN(BSC). SANTIAGODECUBA, Pico Suecia, 1700-1750 m, 7.XII.1069, Muñiz 417c (HAC).

Phyllodon truncatulus (Müll. Hal.) W.R. Buck. GUANTÁNAMO, Baracoa, Arroyo Blanco, 160 m, 19.V.1991, Mustelier y Vicario 12277 (BSC). 
SANTIAGO DE CUBA, Gran Piedra, 1000-1200 m, 7.XII.1970, Borhidi et al. 996 (HAC).

Taxiphyllum scalpellifolium (Müll. Hal.) Broth. SANTIAGO DE CUBA, Alto del Puerto de Boniato, 350 m, 14.V.1979, Pócs 9102/B (BSC).

Taxiphyllum taxirameum (Mitt.) Fleisch. GUANTÁNAMO, Meseta del Guaso, Limonar, cerca del sumidero del Río Cuzco, 400 m, 15.XII.1994, Motito y Potrony 13249 (BSC). SANTIAGODECUBA, Gran Piedra, Jardín La Siberia, 1000-1100 m, 21.V.1979, Pócs y Duany 9115/AC (BSC).

Vesicularia vesicularis (Schwägr.) Broth. var. vesicularis. GUANTÁNAMO, Meseta de Maisí, entre Sabana y elRío Yumurí, pasandopor Santa Rosa, 200m, 15.IV.1998, Motito y Vicario 15571 (BSC).

Vesicularia vesicularis var. crassicaulis (Mitt.) W.R. Buck. SANTIAGODECUBA, Alto de Villalón, $400 \mathrm{~m}$, 12.V.1979, Pócs y Reyes 9101/E (BSC).

Vesicularia vesicularis var. portoricensis (Brid.) W.R. Buck. GUANTÁNAMO, Yunque de Baracoa, 240-450 m, 26.XI.1978, Pócs y Reyes 9063/AW(BSC). SANTIAGODECUBA, Alto de Villalón, Río Ramona, 500 m, 9.IV.1982, Shaw 5377 (BSC, HAC).

Vesicularia vesicularis var. rutilans (Brid.) W.R. Buck. GUANTÁNAMO, Sierra del Purial, Loma La Farola, valle norte, 480 m, 25.XI.1978, Pócs y Reyes 9062/J (BSC). SANTIAGO DE CUBA, Gran Piedra, $1000 \mathrm{~m}$, 3.IV.1982, Shaw 5133 (BSC, HAC).

\section{HYPOPTERYGIACEAE}

Hypopterygium tamarisci (Sw.) Brid. ex Müll. Hal. GRANMA, La Bayamesa, camino a Arroyo 26, 1500$1600 \mathrm{~m}, 18 . I V .2004$, Sánchezetal. s/n(HAJB). SANTIAGO DE CUBA, Gran Piedra, valle del Río Arenal, 9001000 m, 21.V.1979, Pócs y Reyes 9199/N (BSC).

\section{LEMBOPHYLLACEAE}

Pilotrichella cuspidans Renauld. \& Cardot. GRANMA, camino a Pino del Agua, 1200 m, 13.XII.1988, Motito y Vicario 6127 (BSC). GUANTÁNAMO, Yunque de Baracoa, 450-540m, 10.XI.1983, Mustelier 10679(BSC). Pilotrichella flexilis (Hedw.) Ångstr. GRANMA, cima de Pico El Gigante, 1352 m, 7.IX.2004, Rivera 18491 (BSC). SANTIAGO DE CUBA, Gran Piedra, Pico Turquino, 1800m, 17.IV.1987, Vicario 9989(BSC).

\section{LESKEACEAE}

Haplocladium microphyllum (Hedw.) Broth. GUANTÁNAMO, Piedra La Vela, 600 m, 10.XII.1987, Motito et al. 15712 (BSC). SANTIAGODECUBA, Gran Piedra, 900 m, 8.IV.1982, Shaw 5369 (BSC, HAC).

\section{LEUCOBRYACEAE}

Bryohumbertia filifolia (Hornsch.) Frahm var. filifolia. GUANTÁNAMO, Alto de Iberia, 200-540 m, 27.II.1998, Motito 15862 (BSC). HOLGUÍN, Reserva de Jaguaní, La Melba, La Mercedita, Arroyo Jaguará, 200-400 m, 29.VI.1987, Mustelier 8292 (BSC).

Campylopus arctocarpus (Hornsch.) Mitt. var. arctocarpus. HOLGUÍN, Sierra de Nipe, Cuchuflí, 640720 m, 30.IV.1997, Motito y Vicario 14896 (BSC).

*Campylopus carolinae Grout. SANTIAGODECUBA, Sierra Cristal, cañada en el caminoa La China, 23.III.2001, Motito et al. 17012 (BSC).

Campylopus cubensis Sull. HOLGUÍN, Sierra de Nipe, La Micro Onda, 700 m, 26.IV.1997, Motito y Vicario 15028(BSC). SANTIAGO DECUBA, Loma del Gato, 1100 m, 1932, Clemente 345 (HAC).

Campylopusflexuosus (Hedw.) Brid. GUANTÁNAMO, Baracoa, Tabajó, 200 m, 30.III.1988, Motito 6482 (BSC). SANTIAGO DE CUBA, Sierra Cristal, bosque frente al albergue La China, 600-650 m, 10.XI.1989, Motito et al. 10278 (BSC).

Campylopus fragilis (Brid.) B.S.G. subsp. fragilis. GUANTÁNAMO, Baracoa, Tabajó, $100 \mathrm{~m}$, 13.V.1991, Mustelier y Vicario 12180 (BSC). SANTIAGODECUBA, Sierra Maestra, Gran Piedra, 1200 m, 6.IV.1982, Buck 7772 (BSC, HAC).

Campylopus lamellinervis (Müll. Hal.) Mitt. var. lamellinervis. HOLGUÍN, Revuelta de Los Chinos, 850-980m,25.V.1979, Pócs 9175/AD(BSC). SANTIAGO DE CUBA, Gran Piedra, Alto sobre San Esteban, 850 m, 25.V.1979, Pócs 9119/A (BSC).

*Campylopus lamellinervis var. exaltatus (Müll. Hal.) Frahm. SANTIAGO DE CUBA, Sierra Maestra, Loma del Gato, 900 m, 1936, Acuña 150 (HAC).

Campylopus nivalis (Brid.) Brid. GRANMA, Loma Subida Albear, cerca del Pico Bayamesa, 1300-1400 m, 30.XI.1978, Pócs 9066/AF (BSC). SANTIAGO DE CUBA, Gran Piedra, alrededores de La Mercedita, 800-1000 m, 13.III.1987, Motito y Mustelier 4800 (BSC). Campylopus pilifer (Brid.) Brid. subsp. Pilifer. GUANTÁNAMO, Baracoa, Arroyo Blanco, 120-160m, 15.V.1991, Vicario 12217 (BSC). SANTIAGO DE CUBA, Gran Piedra, 1100-1200 m, 10.XII.1970, Borhidi et al. 1071 (HAC).

*Campylopus richardii Brid. HOLGUÍN, Sierra de Nipe, La Micro Onda, Filial Universitaria, 700 m, 26.IV.1997, Motito y Vicario 14807(BSC). SANTIAGO DE CUBA, Gran Piedra, 1000 m, 06.IV.1982, Shaw 5281 (BSC, HAC). 
Campylopus shawii Wilson. GUANTÁNAMO, Reserva Cupeyal del Norte, 500-600 m, 28.VIII.1986, Mustelier 7533 (BSC). SANTIAGO DE CUBA, Sierra Cristal, camino a Canadá, 650 m, 15.II.1989, Motito et al. 10481 (BSC).

Leucobryum albidum (Brid. ex P. Beauv.) Lindenb. GRANMA, Pico Bayamesa, 1500-1700 m, 17.XII.1988, Motito y Vicario 6165 (BSC). SANTIAGODECUBA, Gran Piedra, 1150 m, 10.XI.1978, Pócs y Reyes 9046/ $A F$ (BSC).

Leucobryum antillarum Schimp. GRANMA, La Bayamesa, firme Chago Wilson, Pico 1587, 13001400 m, 25.II.1987, Mustelier y Vicario 7804 (BSC). HOLGUÍN, Sierra Cristal, camino de Baconal a La Zanja, 350-510 m, 30.III.2004, Motito 18194 (BSC).

Leucobryum crispum Müll. Hal. GUANTÁNAMO, Alto de Iberia, subida, 200-540 m, 27.II.1998, Motito 15841 (BSC). SANTIAGO DE CUBA, Sierra Cristal, Arroyo Manzano, entre La China y Batista, 600-650 m, 9.XI.1989, Motito y Potrony 6433 (BSC).

Leucobryum giganteum Müll. Hal. GUANTÁNAMO, Río Barbudo, Viento Frío, 511 m, 5.VI.2004, Motito et al. 18653 (BSC). HOLGUÍN, Sierra de Nipe, Cuchuflí, 640-700 m, 29.04.1997, Motito y Vicario 14783 (BSC). SANTIAGO DE CUBA, Loma San Juan, 7001000 m, 14.VII.1989, Musteliery Vicario 6313 (BSC). Leucobryum martianum (Hornsch.) Hampe. GUANTÁNAMO, Altode Iberia, alrededores dela laguna, 540-600 m, 1.III.1998, Motito 15848(BSC). HOLGUÍN, Moa, La Melba, cerca de la Cascada Dos Comadres, 400 m, 10.X.1980, Pócs y Reyes 9170/A (BSC).

Leucobryum polakowskyi (Müll. Hal.) Cardot. GRANMA, subida a Pico Bayamesa, 1500-1700 m, 17.XII.1988, Motito y Vicario 6175(BSC). HOLGUÍN, Sierra de Nipe, Cuchuflí, 640-700 m, 29.IV.1997, Motito y Vicario 14784 (BSC).

Microcampylopus curvisetus (Hampe) Giese \& Frahm. SANTIAGO DE CUBA, Gran Piedra, 1000-1200 m, 26.X.1969, Borhidi y Muñiz 3775 (HAC).

\section{LEUCOMIACEAE}

Leucomium strumosum (Hornsch.) Mitt. HOLGUÍN, Sierra de Nipe, La Micro Onda, cañada cerca de la Filial Universitaria, 700 m, 26.IV.1997, Motito y Vicario 14815 (BSC). SANTIAGO DE CUBA, Alto de Villalón, 575 m, 26.I.1985, Mustelier 4789 (BSC).

Rhynchostegiopsisflexuosa (Sull.) Müll. Hal. GRANMA, cañada cerca de Pino del Agua, 1300 m, 16.XII.1988, Motito y Vicario 18889 (BSC). GUANTÁNAMO,
Yunque de Baracoa, 200-450 m, 26.XI.1978, Pócs y Reyes 9063/AL (BSC).

\section{METEORIACEAE}

Barbellopsis trichophora (Mont.) W. R. Buck. GUANTÁNAMO, Piedra La Vela, 500-600 m, 11.XII.1997, Motito et al. 15775 (BSC).

Lepyrodontopsis trichophylla (Hedw.) Broth. GRANMA, Las Lagunitas, ladera norte, $1100 \mathrm{~m}$, 21.IV.1979, Pócs y Duany 9084/AJ (BSC). SANTIAGO DE CUBA, Gran Piedra, 1100 m, 10.XI.1978, Pócs y Reyes 9047/AF (BSC).

Meteorium deppei (Müll. Hal.) Mitt. GRANMA, subida a Pico Bayamesa, 1500-1700 m, 17.XII.1988, Motito y Vicario 7344 (BSC). GUANTÁNAMO, Piedra La Vela, 550-600 m, 12.XII.1997, Motito et al. 15585 (BSC). HOLGUÍN, Sierra de Nipe, Cuchuflí, 640720 m, 29.IV.1887, Motito y Vicario 14852 (BSC).

Meteoriumnigrescens (Hedw.) Mitt. GUANTÁNAMO, Piedra La Vela, 600 m, 10.XII.1997, Motito et al. 15722 (BSC). SANTIAGO DE CUBA, Gran Piedra, Pico Kentucky 1020 m, 26.V.1979, Pócs 9126/B (BSC).

Neodicladiella pendula (Sull.) W.R. Buck. Registro de Buck (1998).

*Pseudotrachypus martinicensis (Broth.) W.R. Buck. SANTIAGO DE CUBA, Gran Piedra, 10001200 m, 25.IX.1970, Reyes 8348 (BSC).

Toloxis imponderosa (Taylor) W.R. Buck. GRANMA, Loma La Sabina, 1200-1300 m, 21.IV.1979, Pócs y Duany 9086/L (BSC). GUANTÁNAMO, Yunque de Baracoa,450-540m, 10.XI.1983, Mustelier 10772 (BSC).

Trachypus viridulus (Mitt.) Broth. SANTIAGO DE CUBA, Alto de Villalón, 575 m, 16.I.1985, Mustelier y Vicario 5895 (BSC).

\section{MNIACEAE}

Epipterygium wrightii (Sull.) Lindb. GRANMA, Loma La Sabina, 1100-1200 m, 19.IV.1979, Pócs y Duany 9085/AD (BSC). GUANTÁNAMO. Baracoa, camino hacia Aguacate, 400 m, 14.VII.1979, Potrony 12476 (BSC).

Plagiomnium rhynchophorum (Hook.) T. J. Kop. GRANMA, Pico Regino, 1600 m, 20.IV.1979, Pócs 9091/K (BSC). SANTIAGO DECUBA, Pico Turquino, 900-1700 m, 10.VI.1936, Acuña 415 (HAC).

Pohlia papillosa (Müll. Hal. ex A. Jaeger) Broth. GRANMA, Loma Subida Albear, cerca del Pico Bayamesa, 1300-1400 m, 30.XI.1978, Pócs 9066/AQ (BSC). SANTIAGO DE CUBA, Pico Turquino, 9001525 m, 10.VI.1936, Acuña 375 (HAC). 


\section{MYRINIACEAE}

Austinia tenuinervis (Mitt.) Müll. Hal. GUANTÁNAMO, Meseta del Guaso, Limonar, camino hacia El Fomento, 300-400 m, 16.XII.1994, Motito y Potrony 13302 (BSC).

\section{NECKERACEAE}

Homalia glabella (Hedw.) B.S.G. GRANMA, Loma La Sabina, 1000 m, 15.IV.1979, Pócs y Duany 9077/ $B$ (BSC). GUANTÁNAMO, Viento Frío, camino de Vía Mulata a Arroyo La Pulga (La Gurbia), 740 m, 4.VI.2004, Motito et al. 18584 (BSC). SANTIAGO DE CUBA, Loma del Gato, 500-800 m, 13.I.1987, Mustelier 8875 (BSC).

Homaliodendron flabellatum (Sm.) M. Fleisch. GRANMA,LomaLa Sabina, 1200-1300 m, 21.IV.1979, Pócs 9086/AQ (BSC). SANTIAGO DE CUBA, Alto de Villalón, 575 m, 16.I.1985, Mustelier 4792 (BSC).

Isodrepanium lentulum (Wilson) E. Britton. GRANMA, Pico Bayamesa, 1700m, 15.XI.1986, Musteliery Vicario 7463 (BSC). SANTIAGO DE CUBA, Sierra Cristal, cañada en el camino a La Zanja, 660 m, 23.III.2001, Motito et al. 16948 (BSC).

Neckeropsis disticha (Hedw.) Kindb. GUANTÁNAMO, Viento Frío, camino de Vía Mulata a Arroyo La Pulga (La Gurbia), 740 m, 4.VI.2004, Motito et al. 18587 (BSC). SANTIAGO DE CUBA, Sierra Cristal, San Benito, cafetales de Calabaza, sur de Mayarí Arriba, 300 m, 7.XI.1978, Pócs y Reyes 9044/A (BSC).

Neckeropsis undulata (Hedw.) Reichardt. GRANMA, La Bayamesa, Nuevo Mundo, El Obelisco, 10001050 m, 15.XII.1988, Motito y Vicario 6132 (BSC). SANTIAGO DE CUBA, subida a Monte Biset, 290650 m, 12.V.2004, Motito y Rivera 18228 (BSC).

Orthostichella hexasticha (Schwägr.) W. R. Buck. GRANMA, La Bayamesa, firme Chago Wilson, Pico 1587, 1300-1400 m, 25.II.1987, Musteliery Vicario 6256 (BSC). GUANTÁNAMO, Meseta del Guaso, cerca de Dos Mujeres, 700-750 m, 21.XI.1980, Pócs y Caluff $s / n$ (BSC).

Orthostichellapentasticha (Brid.)W.R. Buck. GRANMA, Subida a Pico Bayamesa, 1300-1500 m, 21.VI.2003, Potrony 17864 (BSC). SANTIAGO DE CUBA, Gran Piedra, 1000 m, 2.IV.1982, Shaw 5015 (BSC, HAC).

Pinnatella minuta (Mitt.) Broth. SANTIAGO DE CUBA, Sierra Maestra, oeste de Matías, farallón de Pozo Azul, 340-380 m, 22.X.1969, Borhidi y Muñiz $s / n$ (HAC).

Porotrichodendron lindigii (Hampe) W. R. Buck. SANTIAGO DE CUBA, Gran Piedra, La Isabelica, 1130 m, 21.V.1979, Pócs y Duany 9112/B (BSC).
Porotrichodendron superbum (Taylor) Broth. SANTIAGODECUBA, Gran Piedra, 1000m, 6.IV.1982, Buck 7722, 7770 (BSC, HAC).

Porotrichum korthalsianum (Dozy \& Molk.) Mitt. GRANMA, La Bayamesa, firme Pico 1555 a Arroyo 26, 1450-1500 m, 20.III.1987, J. Reyes y Caluff 8042 (BSC). SANTIAGO DE CUBA, Gran Piedra, después de La Isabelica, 1000 m, 11.II.2000, Motito et al. 16582 (BSC).

Porotrichum mutabile Hampe. GRANMA, camino entre Pico Botella y Barrio Nuevo, 1300-1400 m, 3.II.2004, Caluff 18104 (BSC). SANTIAGO DE CUBA, Gran Piedra, 1000 m, 2.IV.1982, Shaw 5016(BSC, HAC).

Thamnobryum fasciculatum (Hedw.) I. Sastre GRANMA, Loma Subida Albear, cerca de Pico Bayamesa, 1300-1400 m, 30.XI.1978, Pócs s/n (BSC). SANTIAGO DE CUBA, Gran Piedra, 1000-1200 m, 4.IV.1982, Shaw 5188 (BSC, HAC).

\section{ORTHODONTIACEAE}

Orthodontium pellucens (Hook.) B.S.G. in Müll. Hal. GUANTÁNAMO, Meseta del Guaso, Limonar, cerca del sumidero del Río Cuzco, 400 m, 15.XII.1994, Motito y Potrony 13240 (BSC).

Hymenodon aeruginosus (Hook. f. \& Wilson) Müll. Hal. var. aeruginosus. SANTIAGO DE CUBA, Gran Piedra, 1130m, 10.XI.1978, Pócs y Reyes 9047/AG(BSC).

Hymenodon aeruginosus var. clementii Thér. SANTIAGO DE CUBA, Pico Turquino, 10.VI.1936, Acuña 402 (HAC).

\section{ORTHOTRICHACEAE}

Groutiella apiculata (Hook.) H. A. Crum \& Steere. GUANTÁNAMO, Sierra del Purial, Loma Cotilla, 500 m, 25.XI.1978, Pócs y Reyes 9061/P (BSC). SANTIAGO DECUBA, Gran Piedra, Pico Kentucky, 1000 m, 5.IV.1982, Buck 7671 (BSC, HAC).

Groutiella chimborazense (Spruce ex Mitt.) Florsch.de Waard subsp. Chimborazense. SANTIAGO DE CUBA, Gran Piedra, cima, 1200 m, 10.XI.1978, Pócs y Reyes 9046/AQ (BSC).

Groutiella husnotii (Besch.) H. A. Crum \& Steere. SANTIAGO DE CUBA, Gran Piedra, 1100 m, 7.XII.1970, Borhidi et al. 1065 (HAC).

Groutiella tumidula (Mitt.) Vitt. SANTIAGO DE CUBA, Sierra Cristal, Pico Cristal, $1231 \mathrm{~m}$, 28.III.2001, Trapero 17039 (BSC).

Groutiella wagneriana (Müll. Hal.) H.A. Crum \& Steere. SANTIAGO DECUBA, Pico Turquino, 18001972 m, 6.XII.1969, Borhidi et al. 3776 (HAC). 
Macromitrium cirrosum (Hedw.) Brid. var. cirrosum. GUANTÁNAMO, Sierra del Purial, Loma Cotilla, 500 m, 25.XI.1978, Pócs y Reyes 9061/M (BSC). SANTIAGO DE CUBA, Sierra Cristal, Pico Cristal, 1231 m, 28.III.2001, Trapero 17022 (BSC).

Macromitrium cirrosum var. jamaicense (Mitt.) Grout. HOLGUÍN, Moa, oeste de la Altiplanicie del Toldo, alrededores del Río Piloto, 790 m, 21.IV.2001, Potrony 17471 (BSC). SANTIAGO DECUBA, Gran Piedra, 1100m, 10.XI.1978, Pócs y Reyes 9047/H(BSC).

Macromitrium cirrosum var. stenophyllum (Mitt.) Grout. GRANMA, camino a $5 \mathrm{~km}$ de Pino del Agua, 1200 m, 12.XII.1988, Motito y Vicario 8947 (BSC). GUANTÁNAMO, Alto de Iberia, cañada cerca del Río Iberia, 540 m, 28.II.2001, Motito 16004 (BSC).

Macromitrium harrisii Paris. SANTIAGO DECUBA, Gran Piedra, 1000m, 2.IV.1982, Shaw 5063 (BSC,HAC).

Macromitrium leprieurii Mont. GUANTÁNAMO, Reserva Cupeyal del Norte, 500-600 m, 28.VIII.1986, Mustelier 9543 (BSC). SANTIAGODECUBA, Sierra Cristal, Pico Cristal, 1231 m, 28.III.2001, Trapero 17140 (BSC).

Macromitrium microstomum (Hook. \& Grev.) Schwägr. GRANMA, Pico Bayamesa, 1200-1300, 14.XI.1986, Mustelier y Vicario 9074 (BSC). HOLGUÍN, Cerro Galano, Melones, Arroyo Sao Redondo, 360-400 m, 18.X.1978, Pócs y Caluff 9026/C (BSC).

Macromitrium perichaetiale (Hook. \& Grev.) Müll. Hal. HOLGUÍN, Sierra de Nipe, sur del corte de leña, 600-700 m, 16.X.1941, Morton y Acuña 3923 (HAC).

Macromitrium subperichaetiale Thér. HOLGUÍN, Sagua de Tánamo, alrededores de San Miguel de Cananova, 1946, Natenson 187 (HAC). SANTIAGO DECUBA,LomadelGato,900m, 1935,Acuña9765(HAC).

*Macromitrium swainsonii (Hook.) Brid. SANTIAGO DE CUBA, Pico Suecia, 1700-1750 m, 7.XII.1969, Muñiz 214 (HAC).

*Schlotheimia jamesonii (Arnott) Brid. SANTIAGO DE CUBA, Gran Piedra, alrededores de La Isabelica, 1000 m, 7.IV.1982, Shaw 5349 (BSC, HAC).

Schlotheimia rugifolia (Hook.) Schwägr. GUANTÁNAMO, Piedra La Vela, 600 m, 7.XII.1997, Motito et al. 15616 (BSC). HOLGUÍN, Cerro Galano, sur de Melones, Arroyo Sao Redondo, 200-360 m, 18.X.1978, Pócs y Catasús 9025/G (BSC).

Schlotheimia torquata (Hedw.) Brid. GUANTÁNAMO, Alto de Iberia, camino a la laguna, 540-600 m, 1.III.1998, Motito 15880 (BSC). SANTIAGO DE CUBA, Gran Piedra, detrás de la cafetería, 1000 m, 11.II.2000, Motito et al. 16528 (BSC).

\section{PHYLLOGONIACEAE}

Phyllogonium fulgens (Hedw.) Brid. GRANMA, Buey Arriba, subida entre Alto de La Bandera y Alto del Cojo, 1.VIII.1987, Panfet y Martín 61604(HAJB). SANTIAGO DE CUBA, Sierra Cristal, cabezadas del Río Miguel, Arroyo Manzano, 680 m, 24.III.2001, Motito et al. 17205 (BSC).

*Phyllogonium viride Brid. GUANTÁNAMO, Alto de Iberia, alrededores de la laguna, 540-600 m, 1.III.1998, Motito 15849 (BSC). HOLGUÍN, Sierra Cristal, camino a Palenque, 570 m, 9.V.2001, Motito et al. 17274 (BSC). SANTIAGO DE CUBA, Loma San Juan, 700-1000 m, 14.VII.1989, Motito y Mustelier 16158 (BSC).

Phyllogonium viscosum (P. Beauv.) Mitt. SANTIAGO DECUBA, Gran Piedra, 100-1200, 25.X.1969, Reyes 270 (BSC).

\section{PILOTRICHACEAE}

*Brymela fissidentoides (Hook. f. \& Wilson) W.R. Buck. GUANTÁNAMO, Baracoa, monte Río Quibiján, 30-70 m, 21.V.1991, Musteliery Potrony 12323 (BSC). Callicostella depressa (Hedw.) A. Jaeger. GUANTÁNAMO, Sierra del Purial, Loma La Farola, 480 m, 25.XI.1978, Pócs y Reyes 9062/H (BSC). HOLGUÍN, Sierra de Nipe, Loma La Mensura, 660 m, 13.XI.1978, Pócs y Reyes 9055/AQ (BSC).

* Callicostella pallida (Hornsch.) Ångstr. GRANMA, La Bayamesa, firme Chago Wilson, Pico 1587, 1300-1400 m, 25.II.1987, Mustelier y Vicario 8040 (BSC). SANTIAGO DE CUBA, Gran Piedra, detrás de la cafetería, 1000 m, 11.II.2000, Motito et al. 16561 (BSC).

Callicostella rivularis (Mitt.) A. Jaeger. SANTIAGO DE CUBA, Pico Turquino, 1600-1700 m, 1929, León 11234 (HAC).

Cyclodictyon albicans (Hedw.) Kuntze. GUANTÁNAMO, Meseta del Guaso, Yambeque, Arroyo Luisa, 400 m, 10.V.1995, Motito 13467 (BSC). SANTIAGO DE CUBA, alrededores del Arroyo Almarales, 260-280 m, 13.V.2004, Motito y Rivera 18251 (BSC).

Cyclodictyon albicaule (Besch.) Kuntze. SANTIAGO DE CUBA, Gran Piedra, 1150 m, 10.XI.1978, Pócs y Reyes 9046/M (BSC).

Cyclodictyon bicolor (Besch.) Kuntze. SANTIAGO DE CUBA, Gran Piedra, 1100-1200 m, 19.XII.1974, Reyes 9138 (BSC).

Cyclodictyon subtortifolium (E. B. Bartram) W.R. Buck. GRANMA, Loma Subida Albear, cerca del 
Pico Bayamesa, 1300-1400 m, 30.XI.1978, Pócs 9066/ CC (BSC). GUANTÁNAMO, Yunque de Baracoa, 540 m, 24.VI.1987, Mustelier 8245 (BSC).

Cyclodictyon varians (Sull.) Kuntze. GUANTÁNAMO, Baracoa,Duaba,ElDajao,460-480m, 11.X.1991,Mustelier y Vicario 12807(BSC). SANTIAGODECUBA, Loma del Gato, 890-900 m, 30.III.1987, Mustelier y Vicario 5596 (BSC).

Hookeriopsis luteorufescens (Besch.) A. Jaeger. Registros de Welch (1969) y Buck (1998)

Hypnella leptorrhyncha (Hook. \& Grev.) A. Jaeger. SANTIAGO DE CUBA, Pico Suecia, 1700-1750 m, 7.XII.1969, Muñiz 413 (HAC).

Hypnellapallecens (Hook.) A. Jaeger. GUANTÁNAMO, Yunque de Baracoa, 200-450 m, 26.XI.1978, Pócs y Reyes 9063/AH (BSC). SANTIAGO DE CUBA, Sierra Cristal, albergue de La China, 600-650 m, 11.XI.1989, Motito et al. 10279 (BSC).

Lepidopilidium portoricense (Müll. Hal.) H.A. Crum \& Steere. GUANTÁNAMO, Sierra del Purial, norte de Imías, noreste del Puntón del Mate, 1067 m, 1943, Bucher 457 (HAC).

Lepidopilum amplirete (Sull.) Mitt. GUANTÁNAMO, Meseta del Guaso, Limonar, cerca del sumidero del Río Cuzco, 400 m, 15.XII.1994, Motito y Potrony 13220 (BSC). HOLGUÍN, Moa, Altiplanicie del Toldo, 900-1000 m, 12.X.1980, Pócs 9176/BB (BSC).

*Lepidopilum brevipes Mitt. SANTIAGO DECUBA, Gran Piedra, Pico Kentucky, 1000 m, 5.IV.1982, Buck 7685 (BSC, HAC).

Lepidopilum longifolium Hampe. GUANTÁNAMO, Yunque de Baracoa, 540 m, 24.VI.1987, Mustelier 8234 (BSC). SANTIAGO DE CUBA, Gran Piedra, alrededores de La Isabelica, 1100 m, 26.V.1979, Pócs 9128/AE (BSC).

Lepidopilum polytrichoides (Hedw.) Brid. GRANMA, Loma La Sabina, 1200-1300 m, 21.IV.1979, Pócs y Duany 9086/V (BSC). GUANTÁNAMO, Yunque de Baracoa, 540 m, 24.VI.1987, Mustelier 8236 (BSC).

Lepidopilum scabrisetum (Schwägr.) Steere. GRANMA, cañada cerca de Pino del Agua, 1300 m, 16.XII.1988, Motito y Vicario 9805 (BSC). GUANTÁNAMO, Yunque de Baracoa, 200-450 m, 26.XI.1978, Pócs y Reyes 9063/BN (BSC).

Pilotrichidium antillarum Besch. GUANTÁNAMO, Meseta del Guaso, entre Yambeque y Puente Cubierta, 420-580 m, 9.V.1995, Motito y Vicario 13985 (BSC). SANTIAGO DE CUBA, Pico Turquino, 900-1525 m, 10.VI.1936, Acuña 354 (HAC).
Pilotrichum affine (Hook.) Brid. GRANMA, Pino del Agua, 1300 m, 16.XII.1988, Motito y Vicario 9415 (BSC). SANTIAGODECUBA, Gran Piedra, $1000 \mathrm{~m}$, 2.IV.1982, Buck 7552 (BSC, HAC).

Pilotrichum bipinnatum (Schwägr.) Brid. GUANTÁNAMO, Yunque de Baracoa, 450-540 m, 26.XI.1978, Pócs y Reyes 9064/AB (BSC).

Pilotrichum compositum (Hedw.) P. Beauv. GUANTÁNAMO, Meseta del Guaso, Yambeque, 550580 m, 10.V.1995, Motito y Vicario 13451 (BSC). SANTIAGO DE CUBA, entre Pico Palma Mocha y Las Lagunitas, 1200-1300 m, 16.IV.1979, Pócs y Duany 9079/Q (BSC).

Pilotrichum cristatum Mitt. GUANTÁNAMO, Yunque de Baracoa, 450-540 m, 10.XI.1983, Mustelier 5331, 8272 (BSC).

Pilotrichum evanescens (Müll. Hal.) Crosby. GUANTÁNAMO, Meseta del Guaso, Yambeque, 550-580 m, 10.V.1995, Motito y Vicario 14047 (BSC). SANTIAGO DE CUBA, Alto de Villalón, 575 m, 16.I.1985, Mustelier 5889 (BSC).

Pilotrichum lophophyllum Sull. GUANTÁNAMO, Meseta del Guaso, San Esteban, 650 m, 20.XII.1994, Lobaina 13261 (BSC). SANTIAGO DE CUBA, Gran Piedra, 1000 m, 4.IV.1982, Buck 7621 (BSC, HAC).

Stenodictyon pallidum E. Britton ex H.A. Crum \& Steere. GRANMA, La Bayamesa, firme Chago Wilson, Pico 1587, 1300-1400 m, 25.II.1987, Musteliery Vicario 7807 (BSC). SANTIAGO DE CUBA, Pico Cuba, 1800 m, 15.IV.1987, Mustelier y Vicario 6333 (BSC).

Thamniopsis incurva (Hornsch.) W.R. Buck. HOLGUÍN, Sierra de Nipe, Cuchuflí, 640-720 m, 30.IV.1997, Motito y Vicario 14907 (BSC). SANTIAGODECUBA, Loma del Gato, 1100 m, 7.VIII.1927, Clemente 1800 (HAC).

Thamniopsis undata (Hedw.) W.R. Buck. Registros de Welch (1969) y Buck (1998).

Trachyxiphium guadalupense (Brid.) W.R. Buck. GRANMA, Pico Bayamesa, vertiente norte, 1700 m, 18.III.1987, Musteliery Vicario 9061 (BSC). SANTIAGO DECUBA, faldas del Pico Turquino, 10.VI.1936, Acuña 291 (HAC).

Trachyxiphium vagum (Mitt.) W.R. Buck. GUANTÁNAMO, Alto de Iberia, camino a la laguna, 540-600 m, 1.III.1998, Motito 15871 (BSC).

\section{POLYTRICHACEAE}

Atrichum androgynum (Müll. Hal.) A. Jaeger. GRANMA, camino a Pino del Agua, 1200 m, 13.XII.1988, Motito y Vicario6967(BSC). SANTIAGODECUBA,PicoPalma Mocha, 1380m, 16.IV.1979, Pócs y Duany 9083/N(BSC). 
Atrichum angustatum (Brid.) B.S.G. GRANMA, Pico Bayamesa, 1200-1300 m, 14.XI.1986, Mustelier y Vicario 9063 (BSC). SANTIAGO DE CUBA, Gran Piedra, 1000 m, 2.IV.1982, Buck 7514(BSC, HAC).

Pogonatum campylocarpum (Müll. Hal.) Mitt. GRANMA, Pico Bayamesa, 1500-1700 m, 17.XII.1988, Motito y Vicario 18072 (BSC). Subida de California al Alto de La Gloria, 600-1400 m, 1.VIII.1987, Panfet y Martin 62568 (HAJB).

*Pogonatum pensilvanicum (Hedw.) P. Beauv. GUANTÁNAMO, Baracoa, loma Los Guineos, 19.IV.1986, Arias y Díaz 67536 (HAJB). SANTIAGO DE CUBA, Pico Turquino, falda norte, 900-1700 m, 10.VI.1936, Aсип̃a 372 (HAC).

Pogonatum subflexuosum (Lor.) Broth. GRANMA, Pico Bayamesa, 1500-1700 m, 17.XII.1988, Motito y Vicario 6186 (BSC). SANTIAGO DE CUBA, La Alcarraza, 600-700 m, 21.I.1989, Motito 9457 (BSC).

Pogonatum tortile (Sw.) Brid. GRANMA, Pino del Agua, 1300 m, 16.XII.1988, Motito y Vicario 8892 (BSC). HOLGUÍN, Moa, La Melba, Arroyo Bueno, 170 m, 18.IX.1997, Potrony 15242 (BSC).

Polytrichum juniperinum Hedw. GRANMA, Pico Bayamesa, 1200-1300 m, 17.XII.1988, Motito y Vicario 8924 (BSC). SANTIAGO DE CUBA, La Alcarraza, caminoaLaBayamita,600m,28.I.1989, Motito 9741 (BSC).

\section{POTTIACEAE}

Anoectangium aestivum (Hedw.) Mitt. GUANTÁNAMO, Yunque de Baracoa, 200-450 m, 10.XI.1983, Mustelier 10740 (BSC). SANTIAGO DE CUBA, Gran Piedra, Pico Kentucky, 1000 m, 5.IV.1982, Buck 7667 (BSC, HAC)

Barbula agraria Hedw. GUANTÁNAMO, Meseta de Maisí, Quemados, 200 m, 12.IV.1998, Motito y Vicario 15504 (BSC). SANTIAGO DE CUBA, Sardinero, 20-80 m, 9.IV.1982, Shaw 5371 (BSC, HAC).

Barbula arcuata Griff. GUANTÁNAMO, Meseta de Maisí, entre Sabana y el Río Yumurí, pasando por Santa Rosa, 200 m, 15.IV.1998, Motito y Vicario 15553 (BSC). SANTIAGO DE CUBA, Alto de Villalón, Río Ramona, 500 m, 9.IV.1982, Shaw 5379 (BSC, HAC).

Barbula indica (Hook.) Spreng. var. indica. GRANMA, Cabo Cruz, 10-20 m, 20.I.1979, Catasús 3774 (HAC). GUANTÁNAMO, Meseta de Maisí, Limones, 200-500 m, 9.VII.1989, Motito 11810 (BSC). SANTIAGO DE CUBA, Playa de Siboney, 20 m, 12.XI.1978, Pócs y Reyes 9051/C (BSC).

Dolotortula mniifolia (Sull.) R.H.Zander. HOLGUÍN, orillas del Río Bariay, noreste del central Rafael Freyre,
30 m, 17.X.1978, Pócs 9020/BE (BSC). SANTIAGO DE CUBA, Meseta Santa María del Loreto, orillas de arroyos, 520 m, 18.V.1979, Pócs 9108/H (BSC). Gymnostomiella orcuttii E.B.Bartram. GUANTÁNAMO, Meseta de Maisí, Quemados, 200 m, 14.IV.1998, Motito y Vicario 15837(BSC). SANTIAGODECUBA, Morro de Santiago de Cuba, cuevas en la ladera norte, $30 \mathrm{~m}$, 8.V.1979, Pócs 9100/A (BSC).

Hymenostylium recurvirostrum (Hedw.) Dixon. GUANTÁNAMO, Meseta del Guaso, Limonar, cerca del surgidero del Río Cuzco, 380 m, 17.XII.1994, Motito y Potrony 13320 (BSC). SANTIAGO DE CUBA, La Caoba, 17.X.1989, Motito y Potrony 10081 (BSC).

Hyophila involuta (Hook.) A. Jaeger. GUANTÁNAMO, Meseta del Guaso, cerca de Dos Mujeres, 700-750 m, 21.XI.1980, Pócs y Caluff9183/C (BSC). HOLGUÍN, Banes, Cerro Antonio Barro, oeste de Aldea Filipina de Samá, 360 m, 19.X.1978, Pócs y Borhidi 9030/A(BSC).

Luisierella barbula (Schwägr.) Steere. SANTIAGO DE CUBA, La Maya, Jarahueca, finca Ojo de Agua, 350-400 m, 1935, Acuña 175 (HAC).

*Plaubelia sprengelii (Schwägr.) R.H. Zander var. sprengelii. GUANTÁNAMO, Meseta del Guaso, Limonar, cerca del sumidero del Río Cuzco, 440 m, 15.XII.1994, Motito y Potrony 13204 (BSC).

Plaubelia sprengelii var. stomatodonta (Cardot) R.H. Zander. SANTIAGO DE CUBA, El Avispero, 150-250 m, 8.X.1997, Potrony 15153 (BSC).

*Pleurochaete squarrosa (Brid.) Lindenb. GUANTÁNAMO, Baracoa, Arroyo Blanco, 20-200m, 11.V.1991, Mustelier y Vicario 12145 (BSC).

Pseudosymblepharis schimperiana (Paris) H.A. Crum. GUANTÁNAMO, Yunque de Baracoa, 200-450 m, 26.XI.1978, Pócs y Reyes 9065/AN(BSC). SANTIAGO DE CUBA, Gran Piedra, entre Pico Kentucky y Pico Mogote, 800 m, 25.V.1979, Pócs 9125/D (BSC).

Quaesticula navicularis (Mitt.) R.H. Zander. GUANTÁNAMO, Río Jaibo, Palma, San Juan, 50 m, 1938, Hioram 12898 (HAC).

Splachnobryum obtusum (Brid.) Müll. Hal. GUANTÁNAMO, Meseta de Maisí, Quemados, 200 m, 14.IV.1998, Motito y Vicario 15523 (BSC). SANTIAGO DE CUBA, Alto del Puerto de Boniato, 350 m, 15.VII.1993, Motito y Small 7022 (BSC).

Tortella humilis (Hedw.) Jenn. HOLGUÍN, Nicaro, Levisa, sur-suroeste de Pico Cristal, 18.II.1976, Catasús 2775 (HAC). SANTIAGODECUBA, Alto de Villalón, Río Villalón, 575 m, 24.II.1975, Reyes 5869 (BSC). 
Tortella linearis (Web. \& Mohr.) R.H. Zander. SANTIAGODECUBA, Meseta Santa María del Loreto, orillas de arroyos, 520 m, 18.V.1979, Pócs 9108/V (BSC). Trichostomum fallaciosum Welch \& H.A. Crum. SANTIAGO DE CUBA, Gran Piedra, alrededores de La Isabelica, 1130 m, 21.V.1979, Pócs y Duany 9112/D (BSC).

Trichostomum involutum Sull. GUANTÁNAMO, Yunque de Baracoa, 200-450 m, 1943, Carabia 482 (HAC). SANTIAGO DECUBA, Sierra Cristal, bosque frente al albergue La China, 600-650 m, 11.XI.1989, Motito et al. 10161 (BSC).

Trichostomum subconnivens Thér. GUANTÁNAMO, Meseta de Maisí, Los Llanos, 420 m, 12.VIII.1938, León 18287 (HAC).

Trichostomum tenuirostre var. gemmiparum (Schimp.) R.H.Zander. SANTIAGODECUBA, Gran Piedra, 1000 m, 5.IV.1982, Buck 7714 (BSC, HAC). Weisiopsis spathulifolius H.A. Crum \& E.B. Bartram. GUANTÁNAMO, Baracoa, Tabajó, 200 m, 9.V.1991, Mustelier y Vicario 12035 (BSC).

Weissia controversa Hedw. HOLGUÍN, Sierra de Nipe, Loma de La Bandera, 650 m, 13.XI.1978, Pócs $y$ Reyes 9053/A (BSC). SANTIAGO DE CUBA, La Tabla, 550-650 m, 12.IV.2000, Trapero y Vicario 16611 (BSC).

Weissia jamaicensis (Mitt.) Grout. GUANTÁNAMO, Meseta de Maisí, Gran Tierra, Los Tubos, 400 m, 29.XII.1959, Alaín y López Figueiras 7027 (HAC). HOLGUÍN, Sierra de Nipe, Alto de La Torre. 720780 m, 30.IV.1997, Motito y Vicario 14890 (BSC).

\section{PRIONODONTACEAE}

Prionodon densus (Hedw.) Müll. Hal. GRANMA, Buey Arriba, subida entre Alto de La Bandera y Alto del Cojo, 1.VIII.1987, Panfet 62602 (HAJB). SANTIAGO DE CUBA, Sierra Cristal, Pico Cristal, 500-600 m, 14.II.1989, Motito y Vicario 9780 (BSC).

\section{PTEROBRYACEAE}

Calyptothecium duplicatum (Schwägr.) Broth. GRANMA, Pico Regino, 1600 m, 20.IV.1979, Pócs 9091/AG (BSC). SANTIAGO DE CUBA, entre Pico Palma Mocha y Las Lagunitas, 1200-1300 m, 16.IV.1979, Pócs y Duany 9079/W (BSC).

Henicodium geniculatum (Mitt.) W.R. Buck. GUANTÁNAMO, Piedra La Vela, camino al Arroyo Sonador,550-650m, 6.XII.1997, Motito etal.15649(BSC). SANTIAGO DE CUBA, alrededores del Río Brazo Fuerte, 220 m, 13.V.2004, Motito y Rivera 18262 (BSC).
Hildebrandtiella guyanensis (Mont.) W. R. Buck. GUANTÁNAMO, Meseta del Guaso, La Prenda, norte de GUANTÁNAMO, 250 m, 29.XII.1920, Hioram 4121 (HAC). SANTIAGO DE CUBA, Loma del Gato, 1100 m, 9.XI.1920, Clemente 301 (HAC). Jaegerina scariosa (Lor.) Arzeni. SANTIAGO DE CUBA, Gran Piedra, Arroyo Negro, 1000 m, 5.IV.1982, Buck 7679 (BSC, HAC). La Lata, Brazo Escondido, sur de Filé, 400-500 m, 24.XII.1969, Borhidi y Muñiz $225 f$ (HAC).

*Orthostichopsis tetragona (Sw. ex Hedw.) Broth. GUANTÁNAMO, Yunque de Baracoa, 450-540 m, 26.XI.1978, Pócs y Reyes 9064/A (BSC).

Orthostichopsis tortipilis (Müll. Hal.) Broth. SANTIAGO DE CUBA, Gran Piedra, 1100 m, 5.IV.1982, Buck 7637 (BSC, HAC).

Pireella angustifolia (Müll. Hal.) Arzeni. SANTIAGO DE CUBA, Gran Piedra, valle del Río Arenal, 9001000 m, 21.V.1979, Pócs et al. 9111/G (BSC).

Pireella cymbifolia (Sull.) Cardot. SANTIAGO DE CUBA, La Lata, Brazo Escondido, sur de Filé, 500 m, 25.X.1969, Borhidi y Muñiz 997 (HAC).

Pireella filicina(Hedw.)Cardot.GRANMA,LaBayamesa, firme Chago Wilson, Pico 1587, 1300-1400 m, 25.II.1987, Musteliery Vicario 7822 (BSC). SANTIAGODECUBA, Pico Turquino, 1922, León 11280 (HAC).

*Pireella pohlii (Schwägr.) Cardot. GUANTÁNAMO, Meseta del Guaso, al este del Alto del Mango, $750 \mathrm{~m}$, 23.X.1980, Pócs y Caluff 9198/D (BSC).

Pireella pycnothallodes (Müll. Hal.) M. Fleisch. SANTIAGO DE CUBA, Alto de La Francia, 13001400 m, 21.XII.1986, Musteliery Vicario 7825 (BSC).

\section{PYLAISIADELPHACEAE}

Aptychella proligera (Broth.) Herzog. GRANMA, Pino del Agua, camino a La Bayamesa desde El Nueve, 1372-1400 m, 20.VI.2003, Potrony 17928 (BSC). SANTIAGODECUBA, Pico Turquino, 1300-1800 m, 10.VI.1936, Acuña 338 (HAC).

Heterophyllium acunae Thér. SANTIAGO DECUBA, PicoTurquino, 1200-1800m, 10.VI.1936,Acuña270(HAC). Heterophyllium subpiligerum Thér. SANTIAGO DECUBA, Pico Turquino, 1600-1700 m, 10.VI.1936, Acuña 366 (HAC).

Isopterygium longisetulum (Müll. Hal.) Paris. GUANTÁNAMO, Meseta de Maisí, Santa Rosa, 200 m, 17.IV.1998, Motito y Vicario 15538 (BSC).

Isopterygium subbrevisetum (Hampe)Broth. SANTIAGO DECUBA, Meseta Santa María del Loreto, norte de Yerba de Guinea, 240 m, 27.X.1980, Pócs y Caluff s/ $n$ (BSC). 
Isopterygium tenerum (Sw.) Mitt. GUANTÁNAMO, Meseta del Guaso, Limonar, cerca del surgidero del Río Cuzco, 380 m, 14.XII.1994, Motito y Potrony 13337 (BSC).

Taxithelium planum (Brid.) Mitt. HOLGUÍN, Ramírez, noreste de Melones, 50 m, 16.X.1978, Pócs 9018/B (BSC). SANTIAGO DE CUBA, alrededores del Río Brazo Fuerte, 220 m, 13.V.2004, Motito y Rivera 18258 (BSC).

Taxithelium portoricense R.S. Williams. GUANTÁNAMO, Piedra La Vela, 600 m, 5.XII.1997, Motito et al. 15619 (BSC).

Wijkiaflagellifera (Broth.) H.A. Crum. SANTIAGODE CUBA, Sierra Maestra, Loma del Gato, 800-1100 m, s.d., Hioram 12480 (HAC).

\section{RACOPILACEAE}

Racopilum tomentosum (Hedw.) Brid. GUANTÁNAMO, Yunque de Baracoa, 450-540 m, 10.XI.1983, Mustelier 10517 (BSC). SANTIAGO DE CUBA, La Lata, alrededores del Río Babosa, 300 m, 13.V.2004, Motito y Rivera 18243 (BSC).

\section{RHIZOGONIACEAE}

Pyrrhobryum spiniforme (Hedw.) Mitt. GRANMA, Pico Bayamesa, 1300-1500 m, 17.XII.1988, Motito y Vicario 9820 (BSC). GUANTÁNAMO, Viento Frío, cuenca superior del Río Barbudo. 7.VI.2004, Motito et al. 18648 (BSC).

\section{RUTENBERGIACEAE}

Pseudocryphaea domingensis (Spreng.) W.R. Buck. HOLGUÍN, Sierra Cristal, camino de Baconal a La Zanja, 350-510 m, 30.III.2004, Motito 18193 (BSC). SANTIAGO DE CUBA, alrededores del Río Brazo Fuerte, 220 m, 13.V.2004, Motito y Rivera 18263 (BSC).

\section{SEMATOPHYLLACEAE}

Acroporium caespitosum (Hedw.) W.R. Buck. GRANMA, La Bayamesa, Nuevo Mundo, El Obelisco, 1000-1050 m, 15.XII.1988, Motito y Vicario 8801 (BSC). SANTIAGO DE CUBA, Gran Piedra, 1100-1200 m, 29.XII.1987, Motito 8636(BSC). *Acroporium estrellae (Müll. Hal.) W.R. Buck. GRANMA, cañada cerca de Pino del Agua, $1300 \mathrm{~m}$, 16.XII.1988, Motito y Vicario 9843 (BSC). HOLGUÍN, Sierra de Nipe, Cuchuflí, 640-700 m, 29.IV.1997, Motito y Vicario 14800 (BSC).

Acroporium longirostre (Brid.) W.R. Buck. GUANTÁNAMO,PiedraLa Vela,500-600m, 11.XII.1997, Motito et al. 15747 (BSC). SANTIAGO DE CUBA,
Sierra Cristal, Arroyo Manzano, entre La China y Batista, 600-650 m, 10.XI.1989, Motito et al. 10212 (BSC). Acroporium pungens (Hedw.) Broth. HOLGUÍN, Moa, La Melba, cerca de la Cascada Dos Comadres, 400 m, 10.X.1980, Pócs y Reyes 9170/AF (BSC). SANTIAGO DE CUBA, Gran Piedra, 1000 m, 2.IV.1982, Shaw 5072 (BSC, HAC).

*Donnellia commutata (Müll. Hal.) W. R. Buck. HOLGUÍN, Sierra de Nipe, La Micro Onda, 700 m, 26.IV.1997, Motito y Vicario 15037 (BSC). SANTIAGO DE CUBA, Gran Piedra, 1000-1200 m, 6.IV.1982, Buck 7760 (BSC, HAC).

Meiothecium boryanum (Müll. Hal.) Mitt. HOLGUÍN, Sierra de Nipe,LaMicro Onda, 700m, 24.VI.1997, Motito y Vicario 15039 (BSC). SANTIAGO DE CUBA, Dos Bocas, Caja de Agua, cerca de SANTIAGODECUBA, 1936, Clemente 2109 (HAC).

Rhaphidostichium acestrostegium (Sull.) W. R. Buck. GUANTÁNAMO, Alto de Iberia, camino, 540$600 \mathrm{~m}, 1 . \mathrm{III} .1998$, Motito 15881 (BSC). SANTIAGO DE CUBA, Sierra Cristal, Arroyo Manzano, entre La China y Batista, 600-650 m, 10.XI.1989, Motito et al. 10258 (BSC).

Rhaphidostichium schwaneckeanum (Müll. Hal.) Broth. GRANMA, Pico Bayamesa, 1700 m, 15.XI.1986, Mustelier y Vicario 9069 (BSC). SANTIAGO DE CUBA, Gran Piedra, 1000 m, 2.IV.1982, Buck 7520 (BSC, HAC).

Sematophyllum adnatum (Michx.) E. Britton. Registro de Buck (1998)

Sematophyllum cuspidiferum Mitt. HOLGUÍN, Sierra de Nipe, Cuchuflí, 720 m, 28.IV.1997, Motito y Vicario 14825 (BSC). SANTIAGO DE CUBA, Sierra Cristal, camino a Canadá, 650 m, 15.II.1989, Motito et al. 10510 (BSC).

Sematophyllum galipense (Müll. Hal.) Mitt. GRANMA, camino a Las Canarias, Victorino, 570 m, 6.IX.2004, Rivera 18432 (BSC). GUANTÁNAMO, Meseta del Guaso, Limonar, cerca del surgidero del Río Cuzco, 400 m, 17.XII.1994, Motito y Potrony 13390 (BSC).

Sematophyllum subpinnatum (Brid.) E. Britton. GUANTÁNAMO, Meseta del Guaso, Limonar, cerca del surgidero del Río Cuzco, 380 m, 17.XII.2004, Motito y Potrony 13383 (BSC). SANTIAGO DE CUBA, Gran Piedra, 1000 m, 6.IV.1982, Buck 7718 (BSC, HAC).

Sematophyllum subsimplex (Hedw.) Mitt. GUANTÁNAMO, Meseta del Guaso, Limonar, camino hacia San Juan, 300 m, 16.XII.1994, Motito y Potrony 13934 (BSC). HOLGUÍN, Sierra de Nipe, Loma La Mensura, 660m, 13.XI.1978,Pócs y Reyes 9055/BB(BSC). 
Sematophyllum swartzii (Schwägr.) Welch \& H.A. Crum. GRANMA, Loma La Sabina, 1100-1200 m, 19.IV.1979, Pócs y Duany 9085/O (BSC). SANTIAGO DE CUBA, Sierra Cristal, cabezadas del Río Miguel, Arroyo Manzano, 680m, 24.III.2001, Motito 17195(BSC). *Sematophyllum tequendamense (Hampe) Mitt. SANTIAGO DE CUBA, Gran Piedra, alrededores de La Isabelica, 1000 m, 7.IV.1982, Shaw 5346 (BSC, HAC).

Trichosteleum sentosum (Sull.) A. Jaeger. GUANTÁNAMO, YunquedeBaracoa,200m,26.XI.1978, Pócs y Reyes 9063/AD (BSC). SANTIAGODECUBA, Sierra Cristal, camino a La Zanja, 700 m, 23.III.2001, Motito et al. 17252 (BSC).

\section{SPHAGNACEAE}

Sphagnum henryense Warnst. HOLGUÍN, Sierra deNipe, Cayo La Plancha, 700 m, 1964, Alaín et al. 8015 (HAC). Sphagnum imbricatum Hornsch. ex Russow. HOLGUÍN, Sagua de Tánamo, Sierra de Santa Catalina, 400 m, 1909, Cowell y Baker 4 (HAC).

Sphagnum macrophyllum Bernh. ex Brid. GUANTÁNAMO, Alto de Iberia, 540 m, 28.II.1998, Motito 16029 (BSC). HOLGUÍN, Moa, La Melba, cerca de la Cascada Dos Comadres, 400 m, 10.X.1980, Pócs y Reyes 9170/Z (BSC).

Sphagnum magellanicum Brid. GUANTÁNAMO, Alto de Iberia, 540 m, 29.II.1998, Motito 15989 (BSC). HOLGUÍN, Sierra de Nipe, La Presita, detrás de la Academia, 650 m, 7.V.1997, Motito y Vicario 15011 (BSC).

Sphagnum meridense (Hampe) Müll. Hal. GRANMA, Buey Arriba, Alto del Rondón, 22.V.1988, Panfet 65077 (HAJB). GUANTÁNAMO, Alto de Iberia, 540 m, Álvarez y Berazaín s/n (HAJB). SANTIAGO DECUBA, Sierra Cristal, camino a La Zanja, 680 m, Motito et al. 16996 (BSC).

Sphagnum palustre L. SANTIAGO DECUBA, Sierra Cristal, alrededores y afluentes del Río Miguel, $500 \mathrm{~m}$, 25.III.2001, Potrony y Trapero 17043 (BSC).

Sphagnum perichaetiale Hampe. GUANTÁNAMO, Alto de Iberia, 540 m, 28.II.1998, Motito 16028(BSC). HOLGUÍN, Revuelta de Los Chinos, ladera noreste, 850-980 m, 12.X.1980, Pócs 9175/A (BSC).

Sphagnum portoricense Hampe. GUANTÁNAMO, Alto de Iberia, 540 m, 28.II.1998, Motito 15900, 16030, 16059, 16082, (BSC).

Sphagnum recurvum $\mathrm{P}$. Beauv. SANTIAGODECUBA, Loma del Gato, 1100 m, 1935, Clemente 2002 (HAC). * Sphagnum strictum Sull. SANTIAGO DE CUBA, Gran Piedra, 1000-1200 m, 4.IV.1982, Buck 7622, 7623 (BSC, HAC).

\section{STEREOPHYLLACEAE}

Entodontopsis leucostega (Brid.) W.R. Buck \& Irel. GUANTÁNAMO, Meseta del Guaso, Limonar, camino hacia San Juan, 300 m, 16.XII.1994, Motito y Potrony 13960 (BSC).

Eulacophyllum cultelliforme (Sull.) W.R. Buck \& Irel. GUANTÁNAMO, Meseta del Guaso, entre Yambeque y Puente Cubierta, 420-580m, 9.V.1995, Motito y Vicario 13919 (BSC). SANTIAGO DE CUBA, La Lata, Brazo Escondido, sur de Filé, 500 m, 24.X.1969, Muñiz y Borhidi 237 (HAC).

Stereophyllum radiculosum (Hook.) Mitt. HOLGUÍN, Banes, Cerro Antonio Barro, oeste de Aldea Filipina de Samá,300m, 19.X.1978, Pócs 9029/L(BSC). SANTIAGO DE CUBA, Gran Piedra, norte de la torre de TV sobre el Retiro, 425 m, 14.V.1979, Pócs 9103/B (BSC).

\section{THUIIDIACEAE}

Cyrto-hypnum involvens (Hedw.) W. R. Buck \& H. A. Crum. GUANTÁNAMO, Viento Frío, camino de Vía Mulata a Arroyo La Pulga (La Gurbia), 740 m, 04. VI.2004, Motito et al. 18588 (BSC). SANTIAGO DE CUBA, Gran Piedra, alrededores de La Isabelica, 1000 m, 7.IV.1982, Shaw 5340 (BSC, HAC).

Cyrto-hypnum minutulum (Hedw.) W.R. Buck \& H.A. Crum. GRANMA, La Bayamesa, Arroyo Nuevo Mundo, 1000-1050 m, 15.XII.1988, Motito y Vicario 8800 (BSC). HOLGUÍN, Sierra de Nipe, Cuchuflí, 640-720 m, 29.IV.1997, Motito y Vicario 14850 (BSC). Cyrto-hypnum scabrosulum (Mitt.) W.R. Buck \& H. A. Crum. Registro de Buck (1998).

Cyrto-hypnum schistocalyx (Müll.Hal.) W.R. Buck \& H. A. Crum. SANTIAGO DECUBA, La Lata, Brazo Escondido, sur de Filé, 400-500 m, 24.X.1969, Borhidi y Muñiz 209, 223, 247 (HAC).

*Rauiella praelonga (Schimp. ex Besch.) Wijk \& Margad. GUANTÁNAMO, Yunque de Baracoa, 450-540 m, 10.XI.1983, Mustelier 5082, 5085 (BSC). Thuidium delicatulum (Hedw.) A. Schimp. in B.S.G. GRANMA, Loma La Sabina, ladera noroeste, $900 \mathrm{~m}$, 13.IV.1979, Pócs y Duany 9076/W (BSC). GUANTÁNAMO, Piedra La Vela, 600 m, 5.XII.1997, Motito et al. 15681 (BSC). SANTIAGO DE CUBA, Gran Piedra, Alto sobre Ocaña, 900 m, 25.V.1979, Pócs 9122/C (BSC).

Thuidium pseudoprotensum (Müll. Hal.) Mitt. GUANTÁNAMO, Yunque de Baracoa, 540 m, 24.VII.1987, Mustelier 14712 (BSC).

Thuidium urceolatum Lor. GRANMA, La Bayamesa, Arroyo Nuevo Mundo, 1000 m, 12.XII.1988, Motito y Vicario 6130 (BSC). HOLGUÍN, Sierra de Nipe, Cuchuflí, 640-700 m, 29.IV.1997, Motito y Vicario 14859 (BSC). 


\section{Referencias}

Aguirre, C.J. \& Rangel-Ch, J.O. 2005. Species diversity and richness of the mosses of the Colombian Choco Region. Journal of the Hattori Botanical Laboratory 97: 97-116.

Allen, B.H. \& Crosby, M.R. 1986a. Revision of the genus Crossomitrium (Musci: Meteoriaceae) Journal of the Hattori Botanical Laboratory 61: 423-476.

Allen, B.H. \& Crosby, M.R. 1986b. A revision of the genera Pilotrichidium and Diploneuron (Musci: Hookeriaceae) Journal of the Hattori Botanical Laboratory 61: 45-64.

Ardiles, V.; Cuvertino, J. \& Osorio, F. 2008. Guía de campo briófitos de los bosques templados austarles de Chile. Una introducción al estudio de los musgos, las hepáticas y los antocerotes que habitan en los bosques de Chile. Ed. Corporación Chilena de la Madera, Concepción. 168 pp.

Bizot, M. 1965. Contribution a la flore bryologique de Cuba. Bulletin Mensuel de la Société Linnéenne et des Sociétés Botanique de Lyon 34: 305-328.

Bizot, M. 1973. Contribution a la flore bryologique de Cuba, 2. The Bryologist 76: 340-346.

Britton, E.G. 1922. On some mosses from the Blue Mountain of Cuba. The Bryologist 25: 89-90.

Brummitt, R.K. \& Powell, C.E. 1992. Authors of plant names. Royal Botanical Gardens, Kew.

Buck, W.R. 1984. Taxonomic and nomenclatural notes on West Indian Hypnaceae. Brittonia 36: 178-183.

Buck, W.R. 1987a. Notes on Asian Hypnaceae and associated taxa. Memoirs of the New York Botanical Garden 45: 519-527.

Buck, W.R. 1987b. Taxonomic and nomenclatural rearrangement in the Hookeriales with notes on West Indian taxa. Brittonia 39: 210-224.

Buck, W.R. 1988. Another view of familial delimitation in the Hookeriales. Journal of the Hattori Botanical Laboratory 64: 29-36.

Buck, W.R. 1989a. Henicodium replaces Leucodontopsis (Pterobryaceae). The Bryologist 92: 534.

Buck, W.R. 1989b. Miscellaneous notes on Antillean mosses. 2. Rhaphidostichium (Sematophyllaceae) in the New World. Moscosoa 5: 189-193.

Buck, W.R. 1991. Notes on Neotropical Pterobryaceae. Brittonia 43: 96-101.

Buck, W.R. 1994a. Myriniaceae. In: Sharp, A. J.; Crum, H. \&Eckel,P.M. (eds.) The moss flora of Mexico. Memoirs of the New York Botanical Garden 69: 869-871.

Buck, W.R. 1994b. A new attempt at understanding the Meteoriaceae. Journal of the Hattori Botanical Laboratory 75: 51-72.

Buck, W.R. 1998. Pleurocarpous mosses of the West Indies. Memoirs of the New York Botanical Garden 82: $1-387$.

Buck, W.R. \& Ireland, R.R. 1985. A reclassification of the Plagiotheciaceae. Nova Hedwigia 41: 89-125.
Bytebier, B. \& Chaut-Petiot, M. 2007. A preliminary checklist of the bryoflora of the Taita Hills, Kenya. Tropical Bryology 22: 55-66.

Churchill, S.P. \& Linares, E. 1995. Prodromus Bryologiae Novo-Granatensis. Introducción a la flora de musgos de Colombia. Instituto de Ciencias Naturales - Museo de Historia Natural, Facultad de Ciencias, Universidad Nacional de Colombia, Bogotá. Parte I y II. 925p.

Churchill, S.P. \& Salazar Allen, N. 2001. Mosses. In: Gradstein, S.R.; Churchill, S.P. \& Salazar Allen, N. Guide to the bryophytes of tropical America. Memoirs of the New York Botanical Garden 86. Pp. 240-571.

Crosby, M.R. \& Magill, R.E. 1977. A dictionary of mosses. Monographs Systematic Botany from the Missouri Botanical Garden 3: VII + 43.

Crosby, M.R.; Magill, R.E \& Bauer, C.R. 1992. Index of mosses 1963-1989. Monographs Systematic Botany from the Missouri Botanical Garden 42: 1-646.

Crum, H.A. 1972. A taxonomic account of the Erpodiaceae. Nova Hedwigia 23: 201-224.

Crum, H.A. 1994. Dicranella. In: Sharp, A.J.; Crum, H. \& Eckel, P.M. (eds.). The moss flora of Mexico. Memoirs of the New York Botanical Garden 69: 112-116.

Crum, H.A. \& Buck, W.R. 1994. Anomodontaceae. In: Sharp, A.J.; Crum, H. \& Eckel, P.M. (eds.). The moss flora of Mexico. Memoirs of The New York Botanical Garden 69: 680-686.

Dauphin, G. \& Grayum, M.H. 2005. Bryophytes of the SantaElena Peninsula and Islas Murciélago, Guanacaste, Costa Rica, with special attention to neotropical dry forest habitats. Lankesteriana 5: 53-61.

De Luna, E. \& Buck, W.R. 1991. An undescribed species of Braunia (Hedwigiaceae) from the Andean cloud forest. The Bryologist 94: 401-403.

Duarte, P.P. 1982a. Musgos cubanos: su presencia mundial. Acta Botanica Cubana 9: 1-19.

Duarte, P.P. 1982b. Nueva especie de Amblytropis (Musci: Daltoniaceae) de Cuba. Acta Botanica Cubana 13: 1-5.

Duarte, P.P. 1995. De musci cubensibus notulae (in floram novan cubensem studia intendentia). Fontqueria 42: 117-118.

Duarte, P.P. 1997. Musgos de Cuba. Fontqueria 47: 1-717.

Frahm, J.P. 1987. A revised list of the Campylopus species in the world. Bryologische Beitraege 7: 1-117.

Frahm, J.P. 1991. Dicranaceae: Campylopodioideae, Paraleucobryoideae. Flora Neotropica 54: 1-238.

Goffinet, B \& Buck, W.R. 2010. Classification of the Bryophyta. Disponible en <http://www.eeb.uconn. edu/people/goffinet/Classificationmosses.html> Acceso a 1 abril 2010.

Holmgren, P.K. \& Holmgren, N.H. 2001. Index Herbariorum. Taxon 50: 605.

Holmgren, P.K.; Holmgren, N.H. \& Barnet, L.C. (eds.). 1990. Index Herbariorum. Ed. 8. Regnum Vegetabile 
120. New York Botanical Garden, Bronx.

Holz, J.; Schäfer-Verwimp, A.; Allen, B. \& Buck, W.R. 2002. New and noteworthy mosses from Costa Rica. Journal of Bryology 24: 321-324.

Ireland, R.R. 1986. Synopsis of the genus Taxiphyllum for North America. Lindbergia 12: 153-157.

Ireland, R.R. 1992. The moss genus Isopterygium (Hypnaceae) in Latin America. Tropical Bryology 6: 111-132.

Koponen, A. 1981. Splachnobryaceae, a new moss family. Annales Botanici Fennici 18: 123-132.

Lehmann, J.G.Ch. 1834. Novarum et minus cognitarum stirpium pugillus 6: 1-66.

León, Hno. 1933. Catalogue des mousses de Cuba. Annales de Cryptogamie Exotique 6(3-4): 1-50.

Lewis, G.J.; Ingram, J.M. \& Bradfield, J.E. 2004. Diversity and habitat relationships of bryophytes at an ultramafic site in southern British Columbia. Pp. 199-204. In: Boy, R.S.; Baker, A.J.M. \& Proctor, J. (eds.). Ultramafic rocks: their soils, vegetation and fauna. Proceedings of the Fourth International Conference on Serpentine Ecology, La Habana.

Magill, R.E. 1994. Aptychella. In: Sharp, A.J.; Crum, H. \& Eckel, P.M. (eds.). The moss flora of Mexico. Memoirs of the New York Botanical Garden 69: 979.

Manuel, M.G. 1994. Cryphaeaceae. In: Sharp, A.J.; Crum, H. \& Eckel, P.M. (eds.). The moss flora of Mexico. Memoirs of the New York Botanical Garden 69: 693-695.

McFarland, K.D. 1994. Palamocladium. In: Sharp, A.J.; Crum, H. \& Eckel, P.M. (eds.). The moss flora of Mexico. Memoirs of The New York Botanical Garden 69: 910-911.

Menzel, M. 1985. Die Gattung Pogonatum P. Beauv. (Polytrichales, Musci) in Lateinamerika 1. Taxonomie und Geographie von Pogonatum campylocarpum (C. Müll.) Mitt. und P. subflexuosum (Lor.) Broth. Lindbergia 11: 134-140.

Merwin, M.C.; Gradstein, S.R. \& Nadkarni, N.M. 2001. Epiphytic bryophytes of Monteverde, Costa Rica. Tropical Bryology 20: 63-70.

Nishimura, N. \& Ando. H. 1994. Ectropothecium. In: Sharp, A.J.; Crum, H. \& Eckel, P.M. (eds.). The moss flora of Mexico. Memoirs of the New York Botanical Garden 69: 1037-1039.

Ochi, H. 1980. A revision of the Neotropical Bryoideae, Musci (First Part). Journal of the Faculty of Education, Tottori University, Natural Science 29: 49-154.

Ochi, H. 1981. A revision of the neotropical Bryoideae, Musci (Second Part). Journal of the Faculty of Education, Tottori University, Natural Science 30: 21-55.

Ochi, H. 1994. Bryum. Subfamily Bryoideae. In: Sharp, A.J.; Crum, H. \& Eckel, P.M. (eds.). The moss flora of Mexico. Memoirs of the New York Botanical Garden 69: 454-489.

Pursell, R.A. 1994. Fissidentales. In: Sharp, A.J.; Crum, H. \& Eckel,P.M. (eds.). The moss flora of Mexico. Memoirs of the New York Botanical Garden 69: 31-81.

Pursell, R.A. 1999. Taxonomic notes on Neotropical Fissidens III. Addendum II. The Bryologist 102: 125-127.

Pursell, R.A. 2007. Fissidentaceae. Flora Neotropica 101: 1-278.

Pursell, R.A.; Bruggeman Nannenga, M.A. \& Iwatsuki, Z. 1992. Species of Fissidens (Fissidentaceae, Musci) common to the Neotropics, Asia and Africa. Bryobrothera 1: 49-55.

Reese, W.D. 1993. Calymperaceae. Flora Neotropica 58: 1-99.

Salazar Allen, N. 1992. Notas para la revisión de las especies de Octoblepharum del neotrópico. Tropical Bryology 6: 171-179.

Salazar Allen, N. 1993a. Taxonomic results of the BRYOTROP Expedition to Zaire and Rwanda. 16. Leucophanaceae. Tropical Bryology 8: 149-152.

Salazar Allen, N. 1993b. Leucophanaceae. Flora Neotropica 59: 1-9.

Schubert, R. 1978. Beitrag zur Mossflora Kubas. Feddes Repertorium 89: 307-326.

Smith, D.K. 1994. Funariaceae. In: Sharp, A.J.; Crum, H. \& Eckel,P.M. (eds.). The moss flora of Mexico. Memoirs of the New York Botanical Garden 69: 427-442.

Šoltes, R. 2000. Habitats for rare bryophytes in Slovakia. Lindbergia 25: 124-127.

Spessard-Schueth, L. 1994. Meteorium. In: Sharp, A.J.; Crum, H. \& Eckel, P.M. (eds.). The moss flora of Mexico. Memoirs of the New York Botanical Garden 69: 729-732.

Streimann, H. 1993. Barbella trichophora, on older name for B. cubensis (Musci: Meteoriaceae). The Bryologist 96: 223-225.

Sullivant, W. 1861. Musi Cubense, or mosses collected by Charles Wright in the eastern part of the island of Cuba during the years 1856, 1857, and 1858. Proceedings of the American Academy of Arts and Sciences 5: 273-290.

Thériot, I. 1939. Complements au catalogue des mosses de Cuba et revision de plusieurs genres. Memorias de la Sociedad Cubana de Historia Natural "Felipe Poey" 13: 203-222.

Thériot, I. 1940. Complements au catalogue des mosses de Cuba et revision de plusieurs genres. Memorias de la Sociedad Cubana de Historia Natural "Felipe Poey" 14: 369-372.

Thériot, I. 1941. Complements au catalogue des mosses de Cuba et revision de plusieurs genres. Memorias de la Sociedad Cubana de Historia Natural "Felipe Poey" 15: 211-234

Welch, W.H. 1950. A contribution to the bryophytes flora of Cuba. The Bryologist 53: 238-243. 
Welch, W.H. 1969. The Hookeriaceae of Cuba. The Bryologist 72: 93-136.

Wijk, R. van der; Margadant, W.D. \& Florschütz, P.A. 1959-1969. Index Muscorum I. Regnum Vegetabile 17: 1-548; 26: 1-535; 33: 1-529; 48: 1-604; 65: 1922. International Association for Plant Taxonomy, Utrecht.

Williams, R.S. 1919. Archidium cubense, sp. nov. The Bryologist 22: 2.

Williams, R.S. 1921. Hyophila subcucullata sp. nov. The Bryologist 24: 22-23.

Zander, R.H. 1981. Descriptions and ilustrations of Barbula, Pseudocrossidium and Bryoerythrophyllum (p.p.) of Mexico. Cryptogamie Bryologie Lichénologie 2: $1-22$.

Zander, R.H. 1983. A reevaluation of Neohyophila Crum (Pottiaceae). The Bryologist 86: 134-139.

Zander, R.H. 1993. Genera of the Pottiaceae: Mosses of Harsh Environments. Bulletin of the Buffalo Society of Natural Sciences 32: 1-378.

Zander, R.H. 1994. Barbula. In: Sharp, A.J.; Crum, H. \& Eckel, P.M. (eds.). The moss flora of Mexico. Memoirs of the New York Botanical Garden 69: 286-296.

Zündorf, H. J. 1989. Zur Erforschung der Laubmoosflora von Cuba. Wiss. Z. Friedrich-Schiller-Univ. Jena, Mat.-Naturwiss. Reihe 38: 217-230. 